\title{
LIBBY-NOVICK KUMARASWAMY DISTRIBUTION WITH ITS PROPERTIES AND APPLICATIONS
}

\author{
ABDUL SABOOR ${ }^{1, *}$, ZAFAR IQBAL ${ }^{2}$, MUHAMMAD HANIF, MUNIR AHMAD1 \\ ${ }^{1}$ School of social sciences, National College of Business Administration E Economics, Lahore, Pakistan \\ 2Department of Statistics, Government College Satellite Town Gujranwala, Pakistan
}

*Corresponding author: saboorfsd@hotmail.com

ABSTRACT: The Kumaraswamy distribution is one of the most popular probability distributions with applications to real life data. In this paper, an extension of this distribution called the Libby-Novick Kumaraswamy (LNK) distribution is presented which is believed to provide greater flexibility to model scenarios involving skew-normal data than original one. Analytical expressions for various mathematical properties including its cdf, quantile function, moments, factorial moments, conditional momennts, moment generating function, characteristic function, vitality function, information generating function, reliability measures, mean deviations, mean residual function, Bonferroni and Lorenz Curves are derived.The parameters' estimation of LNK distribution is undertaken using the method of maximum likelihood estimation. A simulation study for different values of sample sizes, to assess the performance of the parameters of LNK distribution is provided. For illustration and performance evaluation of LNK distribution three real-life data sets from the field of engineering and science adapted from earlier studies are used. On comparing the results to previously used methods, LNK distribution shows that it can give consistently better fit than other existing important lifetime models. It is found that the LNK distribution is more suitable and useful to study lifetime data.

Received September 1'st, 2020; accepted September 16 ${ }^{\text {th }}$, 2020; published April 12 ${ }^{\text {th }}, 2021$.

2010 Mathematics Subject Classification. 62E17, 05A15.

Key words and phrases. Libby-Novick Kumaraswamy distribution; moment generating function; mean deviation; reliability measures; maximum likelihood estimation.

(C)2021 Authors retain the copyrights of their papers, and all open access articles are distributed under the terms of the Creative Commons Attribution License. 


\section{INTRODUCTION}

Iqbal et al., (2017) [1] defined the Libby-Novick Kumaraswamy (LNK) distribution. The probability density function $(p d f)$ and cumulative distribution function $(c d f)$ are respectively defined as

$$
\begin{aligned}
f(x) & =a b c \frac{x^{a-1}\left(1-x^{a}\right)^{b-1}}{\left[1-(1-c) x^{a}\right]^{1+b}} ; 0<x<1, \quad a, b, c>0 \\
F(x) & =1-\left(\frac{1-x^{a}}{1-(1-c) x^{a}}\right)^{b}
\end{aligned}
$$

The Libby-Novick Kumaraswamy (LNK) distribution is a continuous probability distribution with double-bounded support. It is very similar, in many respects, to the Libby-Novick Beta (LNB, 1982) [2] distribution and Kumaraswamy (Kum, 1980) [3] distribution. The Kum distribution is a two parameter distribution like Beta distribution Where as M.C. Jones (2009) [4] find out numerous benefits of the Kum distribution over classical beta distribution.The Kum distribution is a special case of McDonald's (1984) [5] generalized Beta of the first kind distribution. One key difference between the Kum and Beta distributions is the availability for the former, but not for the latter, of an invertible closed form cumulative distribution function presented by Mitnick (2013) [6].

The two distributions LNK and LNB are very flexible and can take approximately the same shapes and the former distribution is also a generalized distribution of Kum distribution. There are, however, important realistic differences between LNB and LNK distributions. On the one hand, the availability for the LNK, but not for the LNB distribution, of an invertible closedform cumulative distribution function makes the LNK distribution much better suited than the LNB for activities that require the generation of random varieties, in particular simulation modeling and simulation-based model estimation. In contrast, the lack of tractable-enough expressions for the mean and variance of the LNK distribution has stalled its utilization for modeling purposes; in spite of the advantages that the availability of an invertible closed-form cumulative distribution function entails, the LNK distribution may use rather sparingly in the modeling of stochastic phenomena and processes. Libby-Novick (1982) [2] derived two new multivariate probability density functions, which are the generalized forms of beta distribution 
and $\mathrm{F}$ distribution. They proved that these distributions that seem to be suitable in utility modeling. They reproduced the same distributional form in the cases of marginal and conditional distributions. Chen and Novick (1984) [7] used the Libby-Novick (1982) [2] univariate generalized beta distribution as a prior and estimated the parameters of Bernoulli and Binomial distributions. These expressions are the generalized forms of the standard beta class. Ristic et al., (2013) [8] derived new family of skewed distributions such as Libby and Novick's generalized beta exponential distribution and found some useful properties of this family of distributions. Cordeiro et al., (2014) [9] defined a family of distributions, named the Libby-Novick beta family of distributions, which includes the classical beta generalized and exponentiated generators. This extended family gave reasonable parametric fits to real data in several areas because the additional shape parameters controlled the skewness and kurtosis simultaneously. Ali (2019) [10] worked on new form of Libby-Novick (NLN) distribution and explored some properties of NLN distribution. This model was compared with other distributions by fitting them to a real data set. Rashid et al., (2020) [11] derived different entropy measures and characterized Libby-Novick generalized Beta (LNGB) distribution through various methods. Iqbal et al., (2021) [12] derived mathematical properties of LNGB distribution and applied it to modeling on three real data sets. In this paper we derive basic and advanced properties of LNK distribution and find applications of LNK distribution to three real data sets. In Section 2, a detailed remarks about the graphs of pdf, hazard rate function, reverse hazarz rate function and survival function are provided.

Section 2 also contains the derivation and results of some important mathematical properties of LNK distribution. Maximum likelihood estimators of three parameters of LNK distribution are derived in section 3. In section 4, a simulation of the parameters is carried out for different sample sizes. A number of deduced models are shown in section 5. In section 6, LNK distribution is compared with some other models through three data sets. Some concluding remarks are presented in section 7 .

\section{MATHEMATICAL PROPERTIES}

\subsection{Shape properties of the $p d f$}

The LNK density function defined in (1) has real flexibility and it is shown through graphs w.r.t. some different combinations of values of the parameters. 

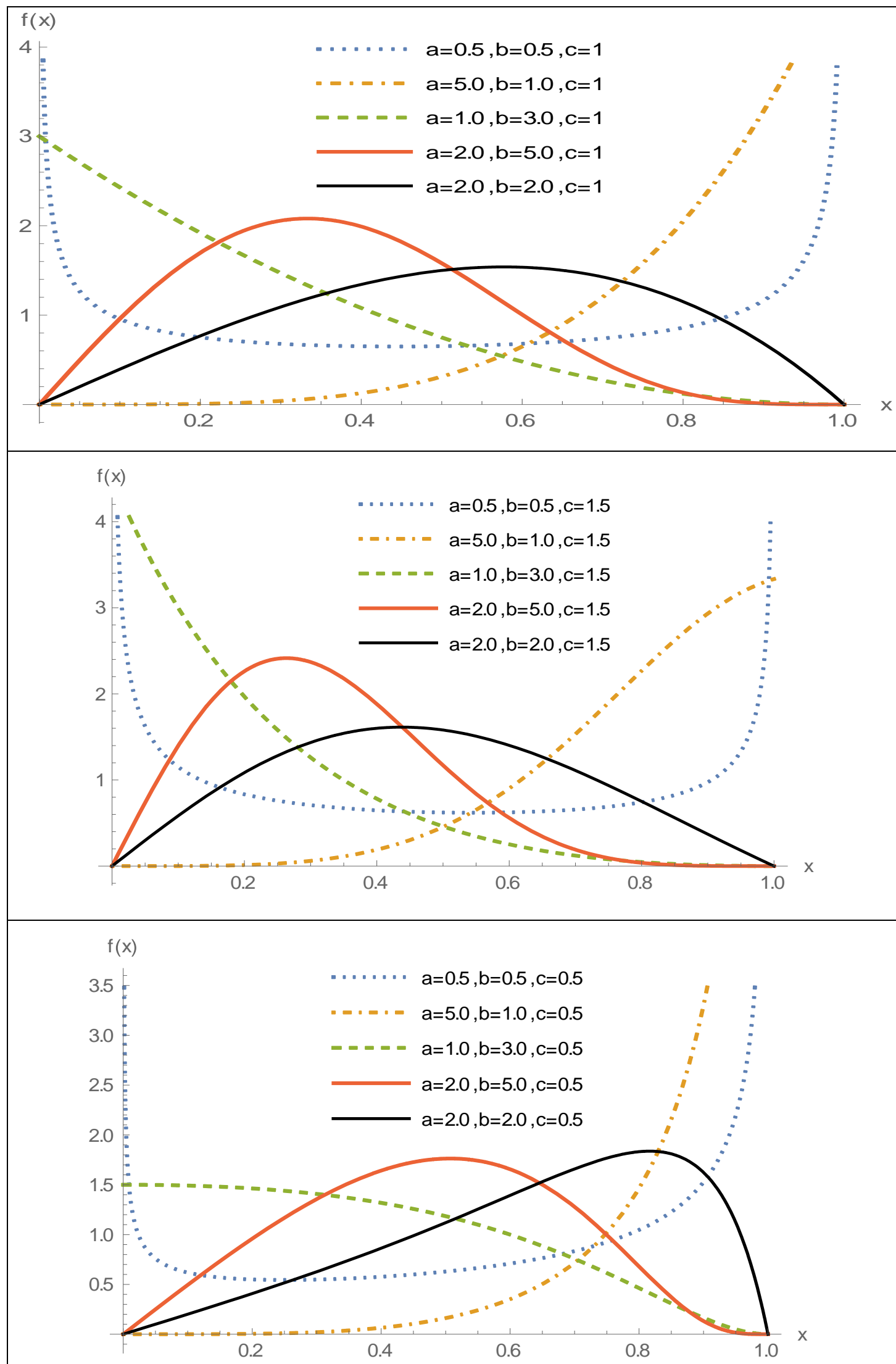


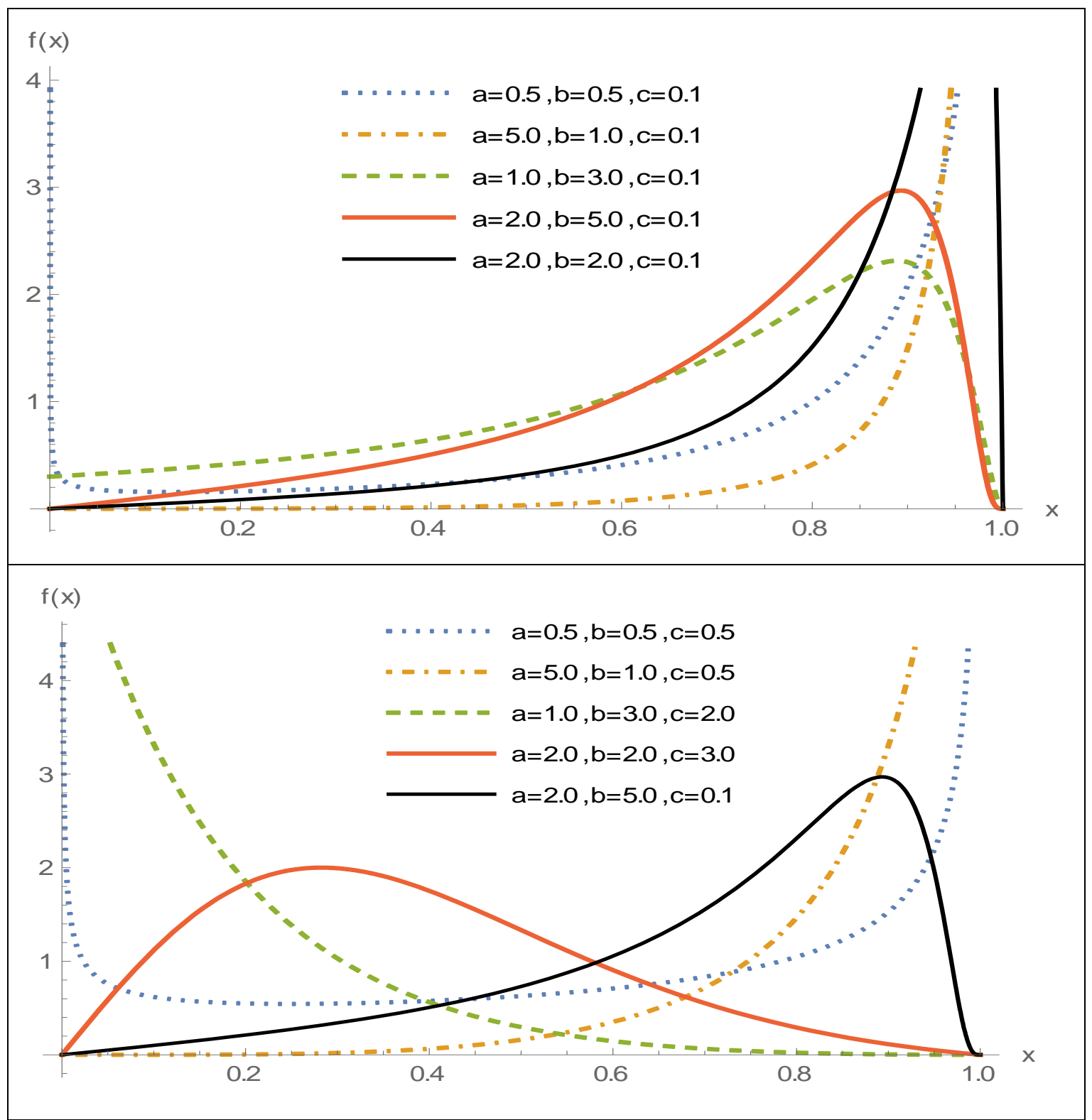

Figure 2.1 $p d f$ Graph of LNK distribution

\section{Shapes of pdf}

(i) For $\mathrm{a}>0, \mathrm{c}>0$ and $0<\mathrm{b}<1$ then LNK distribution is U-shaped.

(ii) For $0<\mathrm{a}<1, \mathrm{~b}>1$ and $0<\mathrm{c}<1$ then LNK distribution is S-shaped.

(iii) For $\mathrm{a}=\mathrm{b}=2, \mathrm{c} \geq 1(\mathrm{c}<1)$ and if $\mathrm{c} \rightarrow \infty(\mathrm{c} \rightarrow 0)$ then LNK distribution increases its positively skewed (negatively skewed) from the symmetric with decreasing mode (increasing mode).

(iv) For $\mathrm{a}=\mathrm{b}=\mathrm{c}=1$, the LNK distribution is uniform distribution.

(v) For $\mathrm{a}>2, \mathrm{~b}>2$ and $\mathrm{c} \geq 1$ the LNK distribution is unimodel positively skewed with decreasing mode when $\mathrm{c} \rightarrow \infty$.

(vi) For $\mathrm{a}>2, \mathrm{~b}=1,0<\mathrm{c}<1$, the form of LNK distribution is an increasing. 
(vii) For $\mathrm{a}>2, \mathrm{~b}=1,1<\mathrm{c}<3.5$, the LNK distribution increase but it increases slowly when $\mathrm{c}$ increases in the interval and for $c \geq 3.5$ the LNK distribution again turns to unimodel.

(viii) For $b=c=1$, the LNK distribution is Power distribution.

(ix) For $\mathrm{a}=\mathrm{c}=1$, the LNK distribution is LNK is a special case of Kum-distribution or reflected exponentiated distribution.

\subsection{Distribution function}

The cumulative distribution function $(c d f)$ of LNK distribution is

$$
F(x)=a b c \int_{0}^{x} \frac{t^{a-1}\left(1-t^{a}\right)^{b-1}}{\left[1-(1-c) t^{a}\right]^{1+b}} d t
$$

By making substitutions $t^{a}=z$ and $u=\frac{c z}{1-z+\mathrm{cz}}$

And after some simplification we have the expression of $c d f$ as shown in (2)

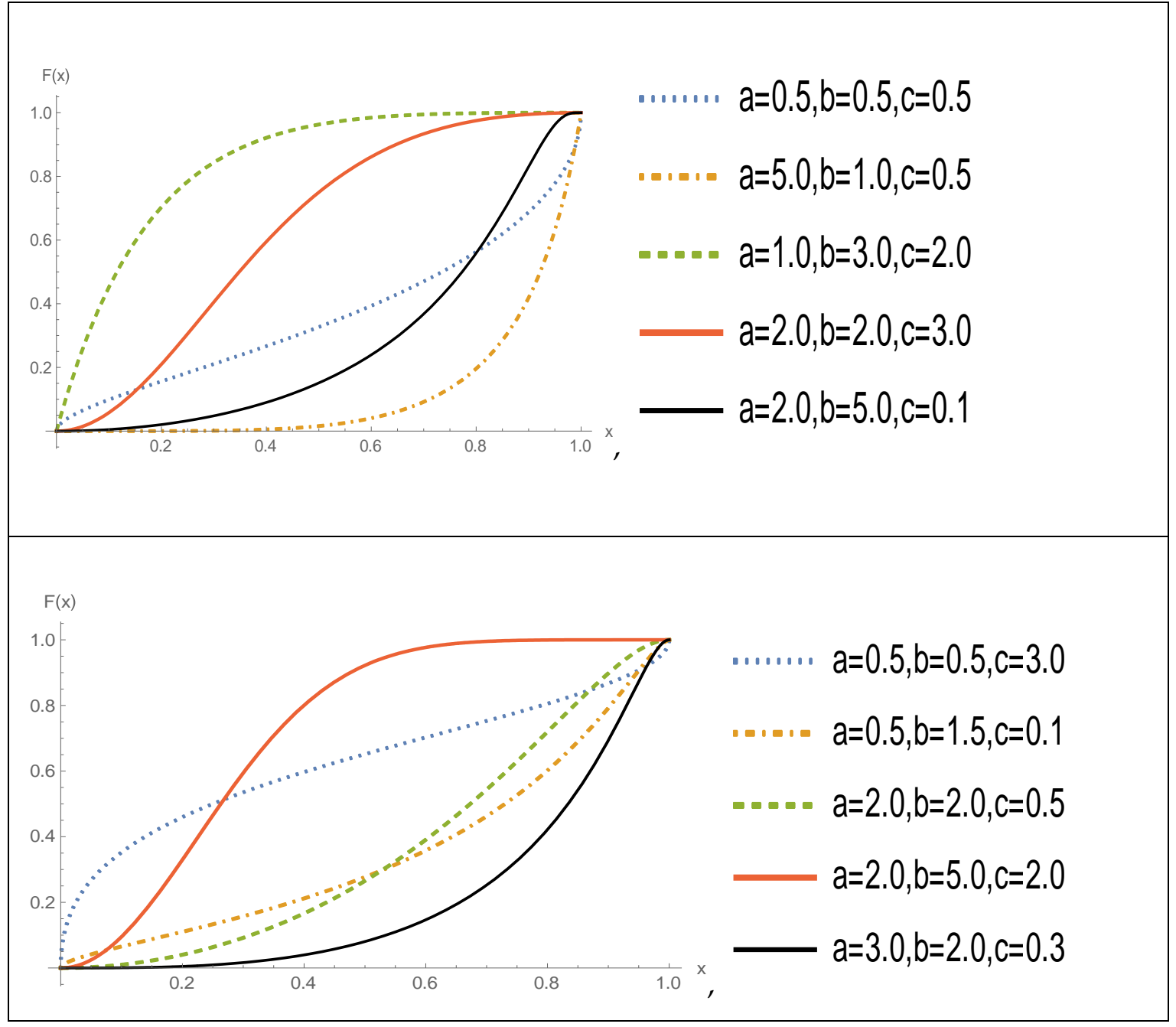

Figure 2.2 cdf Graph of LNK distribution 


\subsection{Quantile Function}

The quantile function of LNK distribution is given by:

$F(x)=p \quad$ Where $0<p<1$

And it $x=F^{-1}(p)$

$1-\left[\frac{1-x^{a}}{1-(1-c) x^{a}}\right]^{b}=p$

After simplification we find the quantile function of the LNK distribution as under;

$$
Q(p ; a, b, c)=\left[\frac{1-(1-p)^{1 / b}}{1-(1-c)(1-p)^{1 / b}}\right]^{1 / a} p \in(0,1)
$$

Where $Q(p ; a, b, c)$ is the inverse of LNK function or quantile of LNK function at $p$. Clearly, the function has explicit form and it can be numerically solved through software for different set of parameters' values. The graph can also be used to illustrate the behaviour of quantile function of LNK distribution.

i. For $a>1, b>1$ by increasing $c$, the quantile value of the LNK distribution decreses comparatively.

ii. For $a<1, c>1$, and for any value of $b$, the quantile value of the LNK distribution increases slowly for $p<0.5$ and for $p>0.5$, the quantile value sharply increases.

iii. For $c \rightarrow 0$, the quantile value of the LNK distribution increases sharply.

\subsection{Hazard Rate Function}

The hazard or instantaneous rate function is denoted by $h(x)$. The hazard function of $x$ can be interpreted as instantaneous rate or the conditional probability density of failure at time $x$, given that the unit has survived until $x$. The hazard rate function of LNK distribution as

$$
h(x ; a, b, c)=\frac{a b c x^{a-1}}{\left[1-(1-c) x^{a}\right]\left(1-x^{a}\right)}
$$




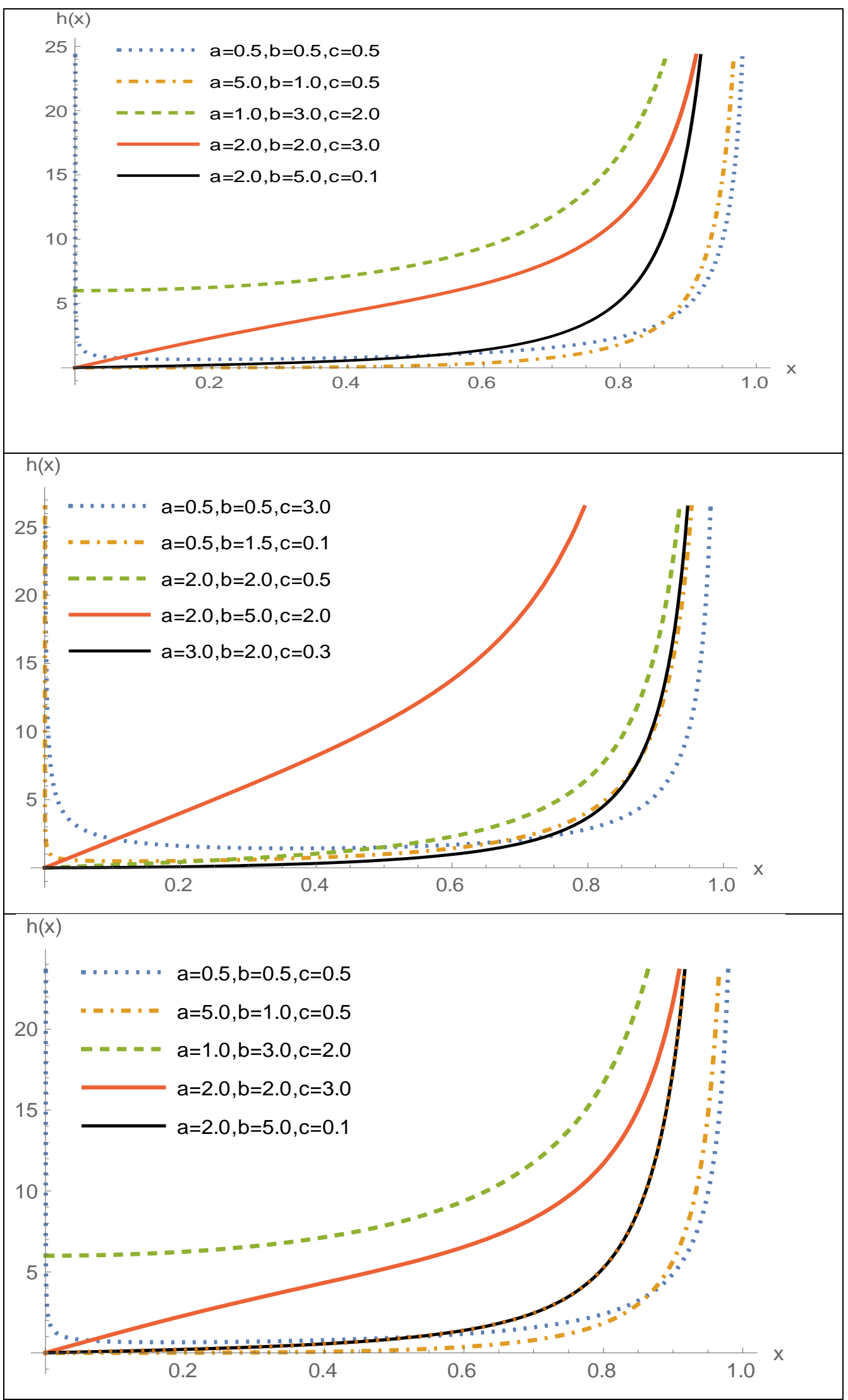

Figure 2.3 hazard rate function Graph of LNK distribution 
The hazard rate function of LNK distribution is of Bath-Tub shape, increasing shape and decreasing shape for specific sets of values of parameters.

\subsection{Reverse Hazard rate function}

The reverse hazard can be interpreted as an approximate probability of failure in $[x, x+\mathrm{d}]$, given that the failure has occurred in $[0, x]$. The reverse hazard function $r(x ; a, b, c)$ of LNK distribution is defined as

$$
r(x ; a, b, c)=\frac{a b c x^{a-1}\left(1-x^{a}\right)^{b-1}}{\left(1-(1-c) x^{a}\right)\left(\left(1-(1-c) x^{a}\right)^{b}-\left(1-x^{a}\right)^{b}\right)}
$$

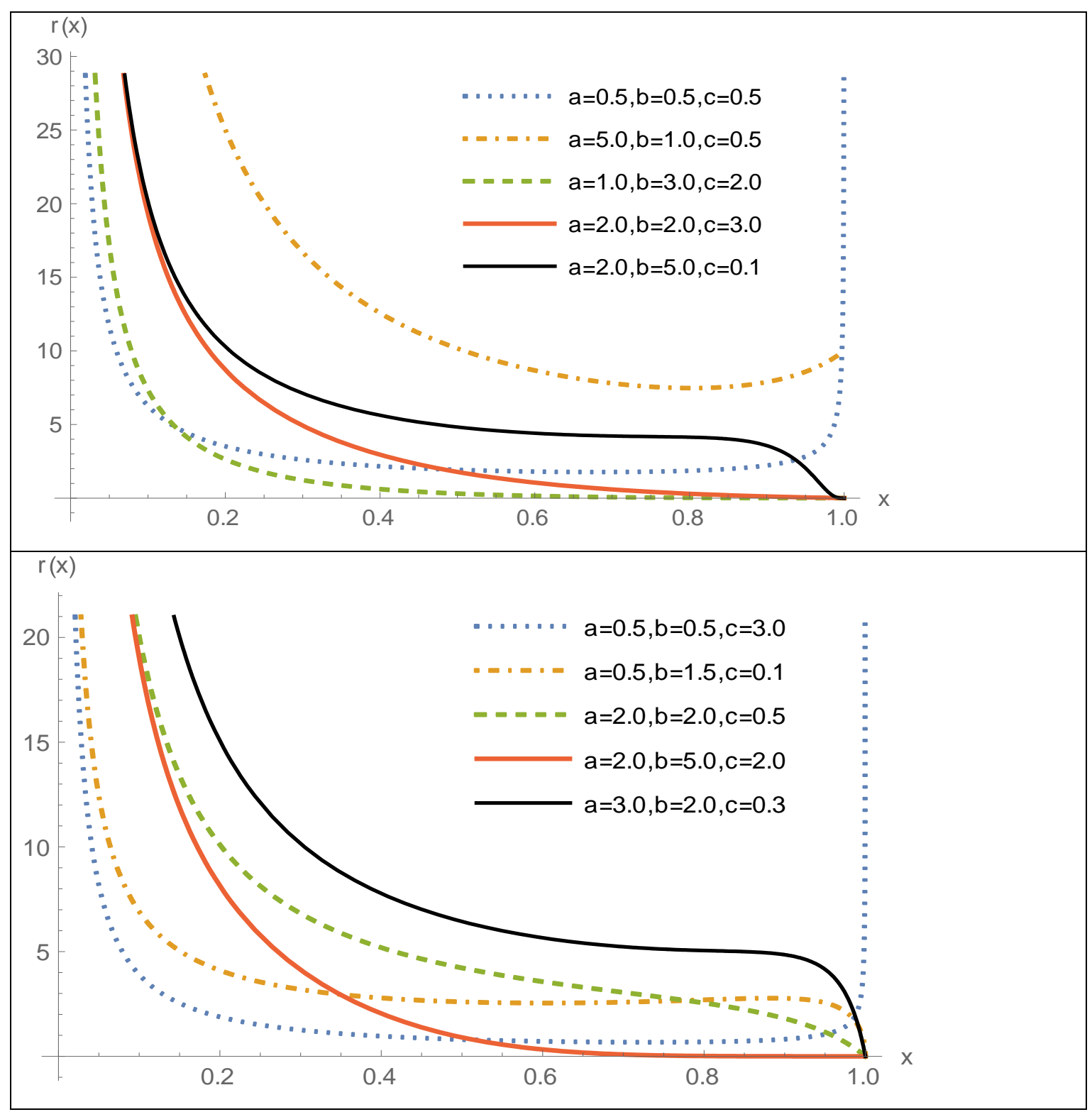

Figure 2.4 reverse hazard rate Graph of LNK distribution 


\subsection{Survival Function}

The survival function or reliability function of LNK distribution is defined as

$$
S(x)=\left[\frac{1-x^{a}}{1-(1-c) x^{a}}\right]^{b}
$$
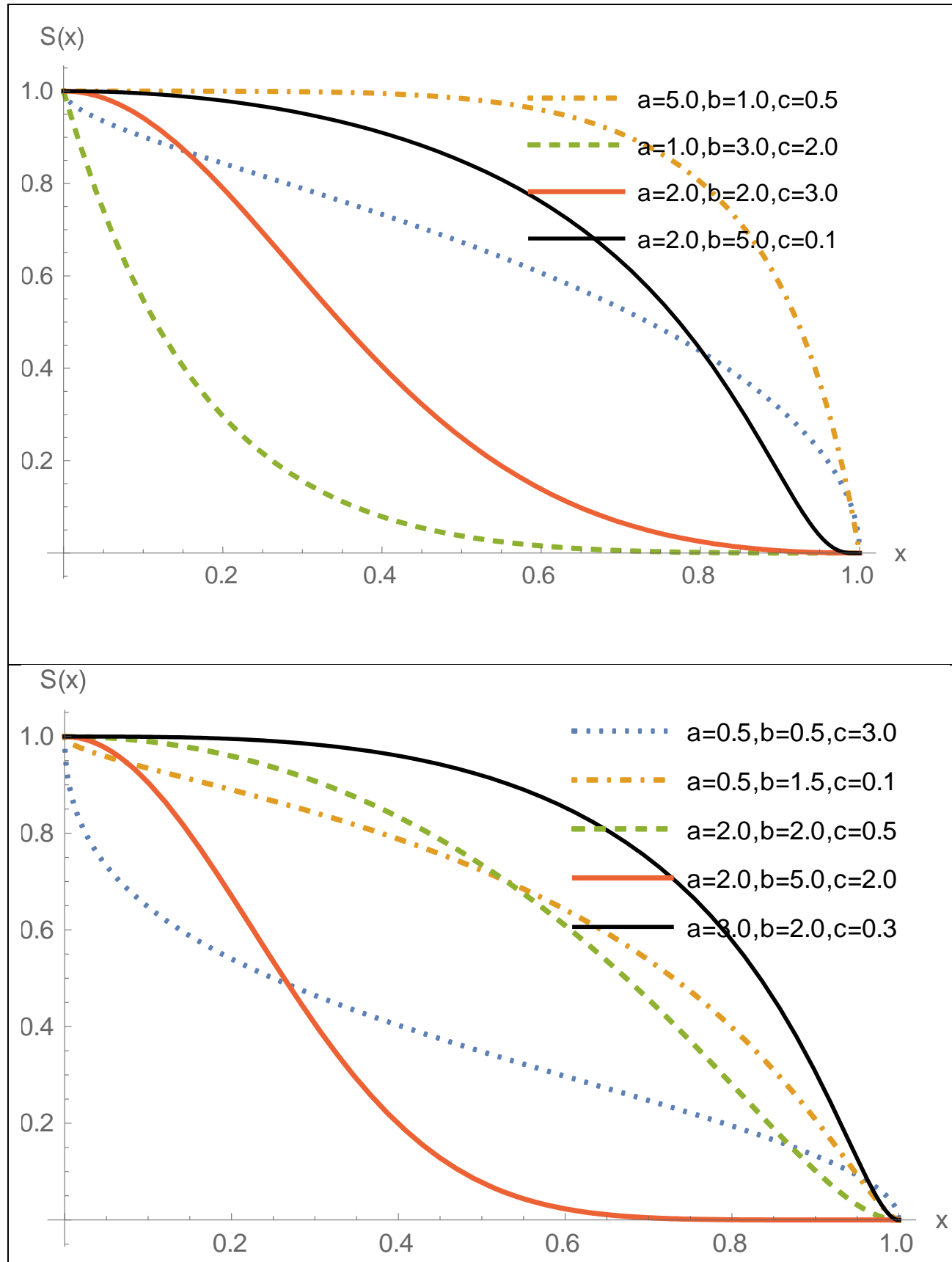

Figure 2.5 survival function Graph of LNK distribution 


\subsection{The $\mathrm{r}^{\text {th }}$ Moment}

The $\mathrm{r}^{\text {th }}$ moments about origion of the LNK distribution is defined as

$$
E\left(x^{r}\right)=\int_{0}^{1} x^{r} f(x) d x
$$

By Substitution (4) becomes

$$
\begin{aligned}
& =\int_{0}^{1} \frac{b c y^{\frac{r}{a}}(1-y)^{b-1}}{[1-(1-c) y]^{1+b}} d y \\
& =b \int_{0}^{1}\left(\frac{u}{c+u-u c}\right)^{\frac{r}{a}}(1-u)^{b-1} d u \\
& =b c^{\frac{-r}{a}} \int_{0}^{1} u^{\frac{r}{a}}(1-u)^{b-1} \sum_{k=0}^{\infty} \frac{\Gamma(r / a+k)}{\Gamma(r / a) k !}\left(1-\frac{1}{c}\right)^{k} u^{k} d u \\
& =b c^{\frac{-r}{a}} \sum_{k=0}^{\infty} \frac{\Gamma(r / a+k)}{\Gamma(r / a) k !}\left(1-\frac{1}{c}\right)^{k} \int_{0}^{1} u^{\frac{r}{a}+k}(1-u)^{b-1} d u \\
& E\left(x^{r}\right)=\frac{b}{c^{r / a}} \sum_{k=0}^{\infty} \frac{\Gamma(r / a+k)}{\Gamma(r / a) k !}\left(1-\frac{1}{c}\right)^{k} B(r / a+k+1, b)
\end{aligned}
$$

\section{Corollary}

$$
\begin{aligned}
& E(x)=\frac{b}{c^{1 / a}} \sum_{k=0}^{\infty} \frac{\Gamma(1 / a+k)}{\Gamma(1 / a) k !}\left(1-\frac{1}{c}\right)^{k} B(1 / a+k+1, b) \\
& E\left(x^{2}\right)=\frac{b}{c^{2 / a}} \sum_{k=0}^{\infty} \frac{\Gamma(2 / a+k)}{\Gamma(2 / a) k !}\left(1-\frac{1}{c}\right)^{k} B(2 / a+k+1, b)
\end{aligned}
$$

\subsection{Moment generating function}

The moment generating function of the LNK distribution about zero is

$$
\begin{aligned}
\mathrm{M}_{x}(\mathrm{t}) & =\mathrm{E}\left(\mathrm{e}^{\mathrm{t} x}\right) \\
& =\sum_{r=0}^{\infty} \frac{b c^{\frac{-r}{a}}}{\Gamma\left(\frac{r}{a}\right)} \sum_{k=0}^{\infty} \Gamma\left(\frac{r}{a}+k\right)\left(1-\frac{1}{c}\right)^{k} B\left(\frac{r}{a}+k+1, b\right) \frac{\left(\mathrm{t}^{r}\right)}{k ! r !} \because \mathrm{e}^{x}=\sum_{r=0}^{\infty} \frac{x^{r}}{r !}
\end{aligned}
$$




\subsection{Factorial Moments}

This section devotes to inceasing and decreasing factorial moments of LNK distribution as under:

\subsubsection{Decreasing Factorial Moments of LNK distribution}

The decreasing factorial moments of the LNK distribution is defined as:

$$
\begin{aligned}
& \mathrm{E}\left[(\mathrm{X})_{\mathrm{n}}\right]=\mathrm{E}[\mathrm{X}(\mathrm{X}-1)(\mathrm{X}-2) \ldots(\mathrm{X}-\mathrm{n}+1)] \\
& =\sum_{\mathrm{r}=0}^{\mathrm{n}} \mathrm{s}(\mathrm{n}, \mathrm{r}) \mathrm{E}\left(\mathrm{X}^{\mathrm{r}}\right) \quad=\sum_{\mathrm{r}=0}^{\mathrm{n}} \sum_{k=0}^{\infty} \frac{s(\mathrm{n}, \mathrm{r})}{\Gamma\left(\frac{r}{a}\right) k !} b^{\frac{-r}{a}} \Gamma\left(\frac{r}{a}+k\right)\left(1-\frac{1}{c}\right)^{k} B\left(\frac{r}{a}+k+1, b\right)
\end{aligned}
$$

Where $s(n, r)$ are the Stirling's numbers of first kind.

\subsubsection{Increasing Factorial Moments of LNBD}

The increasing factorial moments of the LNK distribution is defined as:

$$
\begin{aligned}
\mathrm{E}\left[\mathrm{X}^{(\mathrm{r})}\right] & =\mathrm{E}[\mathrm{X}(\mathrm{X}+1)(\mathrm{X}+2) \ldots(\mathrm{X}+\mathrm{r}-1)] \\
= & \sum_{\mathrm{r}=0}^{\mathrm{n}} d(\mathrm{n}, \mathrm{r}) \mathrm{E}\left(\mathrm{X}^{\mathrm{r}}\right) \\
= & \sum_{\mathrm{r}=0}^{\mathrm{n}} \sum_{k=0}^{\infty} \frac{d(\mathrm{n}, \mathrm{r})}{\Gamma\left(\frac{r}{a}\right) k !} b c^{\frac{-r}{a}} \Gamma\left(\frac{r}{a}+k\right)\left(1-\frac{1}{c}\right)^{k} B\left(\frac{r}{a}+k+1, b\right)
\end{aligned}
$$

Where $d(\mathrm{n}, \mathrm{r})$ can be deduced from the relation

$$
\mathrm{s}(\mathrm{n}, \mathrm{r})=(-1)^{n-r} d(\mathrm{n}, \mathrm{r})
$$

\subsection{Negative Moments}

The negative moments of the LNK distribution is defined as:

$\mathrm{E}\left(\mathrm{X}^{-\mathrm{r}}\right)=\int_{0}^{1} x^{-\mathrm{r}} f(x ; \mathrm{a}, \mathrm{b}) \mathrm{d} x$

By applying the substitution (4), we have

$$
\mathrm{E}\left(\mathrm{X}^{-\mathrm{r}}\right)=b c^{\frac{r}{a}} \sum_{k=0}^{\infty}\left(\begin{array}{l}
n \\
k
\end{array}\right)(-1)^{k}\left(1-\frac{1}{c}\right)^{k} B\left(-\frac{r}{a}+k+1, b\right)
$$




\subsection{Incomplete Moments}

The Incomplete moments of the LNK distribution is defined as:

$$
\begin{aligned}
& \phi_{r}(x)=\int_{0}^{x} x^{r} f(x) d x \\
& =\int_{0}^{x} \frac{x^{r} a b c x^{a-1}\left(1-x^{a}\right)^{b-1}}{\left.\Gamma 1-(1-c) x^{a}\right]^{1+b}} d x \\
& =\frac{b}{c^{\frac{r}{a}}} \sum_{k=0}^{\infty} \frac{\Gamma\left(\frac{r}{a}+k\right)}{\Gamma\left(\frac{r}{a}\right) k !}\left(1-\frac{1}{c}\right)^{k} I\left(\frac{c x^{a}}{1-x^{a}+c x^{a}} ; \frac{r}{a}+k+1, b\right)
\end{aligned}
$$

\subsection{Scaled Total Time for Aging Properties}

The scaled total time of the LNK distribution is defined as:

$$
\begin{aligned}
& S_{F}(F(x))=\frac{1}{\mu} \int_{0}^{x} S(y) d y=\frac{1}{\mu} \int_{0}^{x} \int_{y}^{1} f(t) d t d y \\
& S_{F}(F(x))=\frac{1}{\mu}\left(x-\int_{0}^{x} F(y) d y\right) \\
& S_{F}(F(x))=\frac{1}{\mu} \int_{0}^{x}\left[\frac{1-y^{a}}{1-(1-c) y^{a}}\right]^{b} d y
\end{aligned}
$$

\subsection{Conditional Moments}

The conditional moments of the LNK distribution is defined as:

$$
\begin{aligned}
& \mathrm{M}_{\mathrm{r}}^{\prime}=\mathrm{E}\left[\mathrm{X}^{\mathrm{r}} \mid \mathrm{X}>x\right] \\
& \mathrm{M}_{\mathrm{r}}^{\prime}=\frac{1}{\overline{\mathrm{F}}} \int_{x}^{1} \mathrm{t}^{\mathrm{r}} f(\mathrm{t}) \mathrm{dt}
\end{aligned}
$$

By applying the substitution (4) and after some simplification, we have

$$
\mathbf{M}_{\mathrm{r}}^{\prime}=\frac{\left(1-(1-c) x^{a}\right)^{b}}{\left(1-x^{a}\right)^{b}}\left[\mu_{r}^{\prime}-\frac{b}{c^{\frac{r}{a}}} \sum_{k=0}^{\infty} \frac{\Gamma\left(\frac{r}{a}+k\right)}{\Gamma\left(\frac{r}{a}\right) k !}\left(1-\frac{1}{c}\right)^{k} I\left(\frac{c x^{a}}{1-x^{a}+c x^{a}} ; \frac{r}{a}+k+1, b\right)\right]
$$

\subsection{Mean Residual Function}

The mean residual function of the LNK distribution is defined as: 


$$
\begin{aligned}
\mathrm{m}(x) & =\mathrm{E}[\mathrm{X}-x \mid \mathrm{X}>x] \\
\mathrm{m}(x) & =\mathrm{E}[\mathrm{X} \mid \mathrm{X}>x]-x \\
& =\frac{\left(1-(1-c) x^{a}\right)^{b}}{\left(1-x^{a}\right)^{b}}\left[\mu_{1}^{\prime}-\frac{b}{c^{\frac{1}{a}}} \sum_{k=0}^{\infty} \frac{\Gamma\left(\frac{1}{a}+k\right)}{\Gamma\left(\frac{1}{a}\right) k !}\left(1-\frac{1}{c}\right)^{k} I\left(\frac{c x^{a}}{1-x^{a}+c x^{a}} ; \frac{1}{a}+k+1, b\right)\right]-x
\end{aligned}
$$

\subsection{Vitality Function}

The vitality function of the LNK distribution is defined as:

$$
\begin{aligned}
& \mathrm{V}(x)=\mathrm{E}[\mathrm{X} \mid \mathrm{X}>x] \\
& \mathrm{V}(x)==\frac{\left(1-(1-c) x^{a}\right)^{b}}{\left(1-x^{a}\right)^{b}}\left[\mu_{1}^{\prime}-\frac{b}{c^{\frac{1}{a}}} \sum_{k=0}^{\infty} \frac{\Gamma\left(\frac{1}{a}+k\right)}{\Gamma\left(\frac{1}{a}\right) k !}\left(1-\frac{1}{c}\right)^{k} I\left(\frac{c x^{a}}{1-x^{a}+c x^{a}} ; \frac{1}{a}+k+1, b\right)\right]
\end{aligned}
$$

\subsection{Geometric Vitality Function}

The Geometric vitality function of the LNK distribution is defined as:

$$
\begin{aligned}
& \log (x)=\mathrm{E}[\log \mathrm{X} \mid \mathrm{X}>x] \\
& \left.\begin{array}{rl}
\log \mathrm{G}(x) & =\frac{1}{1-\mathrm{F}(\mathrm{x})} \int_{x}^{1} \log \mathrm{t} f(\mathrm{t}) \mathrm{dt} \\
= & \frac{1}{1-\mathrm{F}(\mathrm{x})}\left(\int_{0}^{1} \log \mathrm{t} f(\mathrm{t}) \mathrm{dt}-\int_{0}^{x} \log \mathrm{t} f(\mathrm{t}) \mathrm{dt}\right) \\
=\frac{1}{1-\mathrm{F}(\mathrm{x})}\left(\left[\frac{1}{a}(\psi(1)-\psi(1+b))-\frac{\ln \mathrm{c}}{a}+b \sum_{i=1}^{\infty} \frac{1}{i}\left(1-\frac{1}{\mathrm{c}}\right)^{i} \mathrm{~B}(i+1, b)\right]-\int_{0}^{x} \log \mathrm{t} f(\mathrm{t}) \mathrm{dt}\right) \\
=\frac{1}{1-\mathrm{F}(\mathrm{x})}\left(\left[\frac{1}{a}(\psi(1)-\psi(1+b))-\frac{\ln \mathrm{c}}{a}+b \sum_{i=1}^{\infty} \frac{1}{i}\left(1-\frac{1}{\mathrm{c}}\right)^{i} \mathrm{~B}(i+1, b)\right]-\frac{b^{1-(1-c) x^{a}}}{a}(1-u)_{0}^{b-1}(\ln u) d u\right. \\
+\frac{b}{a} \int_{0}^{\frac{c x^{a}}{1-(1-c) x^{a}}}(1-u)^{b-1} \ln (c+u-u c) d u
\end{array}\right)
\end{aligned}
$$




\subsection{Characteristics Function}

The characteristic function of the LNK distribution is defined as:

$$
\begin{aligned}
\phi_{x}(\mathrm{t}) & =\mathrm{E}\left(\mathrm{e}^{i \mathrm{tx}}\right) \\
& =\sum_{r=0}^{\infty} \frac{b c^{\frac{-r}{a}}}{\Gamma\left(\frac{r}{a}\right)} \sum_{k=0}^{\infty} \Gamma\left(\frac{r}{a}+k\right)\left(1-\frac{1}{c}\right)^{k} B\left(\frac{r}{a}+k+1, b\right) \frac{(i t)^{r}}{k ! r !} \because \mathrm{e}^{x}=\sum_{r=0}^{\infty} \frac{x^{r}}{r !}
\end{aligned}
$$

\subsection{Information generating function}

The information generating function of the LNK distribution is defined by $P(s)$ and found from

$$
\begin{aligned}
P(s) & =E\left(f^{s-1}\right) \\
& =\int_{0}^{1}\left(\mathrm{abc} \frac{x^{a-1}\left(1-x^{a}\right)^{b-1}}{\left[1-(1-c) x^{a}\right]^{\mathrm{b}+1}}\right)^{s} \mathrm{dx}
\end{aligned}
$$

By applying the substitution (4), we have

$$
\begin{aligned}
P(s) & =a^{s-1} b^{s} c^{(s-1) / a} \sum_{i=0}^{\infty} \frac{\Gamma((a+1)(1-s) / a+i)}{\Gamma((a+1)(1-s) / a) i !}\left(1-\frac{1}{c}\right)^{i} \int_{0}^{1} u^{(a-1)(\mathrm{s}-1) / a}(1-u)^{s(b-1)} u^{i} d u \\
& =a^{s-1} b^{s} c^{(s-1) / a} \sum_{i=0}^{\infty} \frac{\Gamma((a+1)(1-s) / a+i)}{\Gamma((a+1)(1-s) / a) i !}\left(1-\frac{1}{c}\right)^{i} \int_{0}^{1} u^{(a-1)(s-1) / a+i}(1-u)^{s(b-1)} d u \\
& =a^{s-1} b^{s} c^{(s-1) / a} \sum_{i=0}^{\infty} \frac{\Gamma((a+1)(1-s) / a+i)}{\Gamma((a+1)(1-s) / a) i !}\left(1-\frac{1}{c}\right)^{i} B((a-1)(s-1) / a+i+1, s(b-1)+1)
\end{aligned}
$$

\subsection{Some Other Measures of Averages}

\subsubsection{Harmonic Mean}

This section contains Harmonic mean of $x$ for the LNK distribution

The Harmonic mean of $x$ is as under:

$H_{x}=\frac{1}{E\left(\frac{1}{x}\right)}$

Consider $E\left(\frac{1}{x}\right)=\int_{0}^{1} \frac{1}{x} f(x) d x$ 
$=a b c \int_{0}^{1} \frac{x^{a-2}\left(1-x^{a}\right)^{b-1}}{\left[1-(1-c) x^{a}\right]^{1+b}} d x$

By substituting $x^{a}=y$, we have

$=\int_{0}^{1} \frac{b c y^{-\frac{1}{a}}(1-y)^{b-1}}{[1-(1-c) y]^{1+b}} d y$

After substitution (4), and simplification, we have

$$
\begin{aligned}
& =\int_{0}^{1} \frac{b u^{\frac{-1}{a}}(1-u)^{b-1}}{[c+u-u c]^{\frac{-1}{a}}} d u \\
& =\int_{0}^{1} b u^{\frac{-1}{a}}(1-u)^{b-1}(c+u-u c)^{\frac{1}{a}} d u \\
& =b c^{\frac{1}{a}} \int_{0}^{1} u^{\frac{-1}{a}}(1-u)^{b-1} \sum_{i=0}^{\infty}\left(\begin{array}{l}
1 / a \\
i
\end{array}\right)\left[-\left(1-\frac{1}{c}\right) u\right]^{i} d u \\
& E\left(\frac{1}{x}\right)=b c^{\frac{1}{a}} \sum_{i=0}^{\infty}\left(\begin{array}{l}
1 / a \\
i
\end{array}\right)\left[-\left(1-\frac{1}{c}\right)\right]^{i} B\left(\frac{-1}{a}+i+1, b\right) \\
& H_{x}=\frac{1}{b c^{\frac{1}{a}} \sum_{i=0}^{\infty}\left(\begin{array}{l}
1 / a \\
i
\end{array}\right)\left[-\left(1-\frac{1}{c}\right)\right]^{i} B\left(\frac{-1}{a}+i+1, b\right)} \text { for } a>1 \text {. }
\end{aligned}
$$

\section{Corollary}

If $\mathrm{c}=1$, Then $H_{x}=\frac{1}{b B\left(\frac{-1}{a}+1, b\right)}$ is the Harmonic Mean of Kum distribution

\subsubsection{Geometric Mean}

The logarithm of the geometric $G_{X}$ of a distribution with random variable $X$ is the arithmetic mean of $(\ln X)$, or, equivalently, its expected value $\ln G_{X}=E(\ln X)$.

$$
E(\ln x)=\int_{0}^{1}(\ln x) f(x) d x
$$


$E(\ln x)=\int_{0}^{1}(\ln x) \frac{a b c x^{a-1}\left(1-x^{a}\right)^{b-1}}{\left[1-(1-c) x^{a}\right]^{1+b}} d x$

By substitution $x^{a}=y$ and (4), after simplification, we have

$G_{X}=\exp \left[\frac{1}{a}(\psi(1)-\psi(1+b))-\frac{\ln \mathrm{c}}{a}+b \sum_{i=1}^{\infty} \frac{1}{i}\left(1-\frac{1}{\mathrm{c}}\right)^{i} \mathrm{~B}(i+1, b)\right]$

2.19.3 Mode

Mode is obtained by solving $\frac{\mathrm{d}}{\mathrm{d} x} f(x)=0$

$$
f(x)=\frac{\mathrm{c}^{\mathrm{a}} x^{\mathrm{a}-1}(1-x)^{\mathrm{b}-1}}{\mathrm{~B}(\mathrm{a}, \mathrm{b})[1-(1-\mathrm{c}) x]^{\mathrm{a}+\mathrm{b}}}
$$

Mode of LNK distribution is obtained by solving

$$
\begin{aligned}
& \frac{d}{d x} f(x)=0 \\
& f^{\prime}(x)=\left\{\frac{a-1}{x}-\frac{a(b-1) x^{a-1}}{\left(1-x^{a}\right)}-\frac{a(1+b)(c-1) x^{a-1}}{\left[1-(1-c) x^{a}\right]}\right\} f(x) \\
& \frac{a-1}{x}-\frac{a(b-1) x^{a-1}}{\left(1-x^{a}\right)}-\frac{a(1+b)(c-1) x^{a-1}}{\left[1-(1-c) x^{a}\right]}=0
\end{aligned}
$$

Multiplying both sides by $x$

$$
a-1-\frac{a(b-1) x^{a}}{\left(1-x^{a}\right)}-\frac{a(1+b)(c-1) x^{a}}{\left[1-(1-c) x^{a}\right]}=0
$$

Put $x^{a}=y$

$$
\begin{aligned}
& a-1-\frac{a(b-1) \mathrm{y}}{1-y}-\frac{a(1+b)(c-1)}{1-(1-c) y}=0 \\
& (a-1)(1-\mathrm{y})(1-\mathrm{y}+\mathrm{cy})-\{\mathrm{a}(\mathrm{b}-1) \mathrm{y}(1-\mathrm{y}+\mathrm{cy})\}-\{\mathrm{a}(1+\mathrm{b})(\mathrm{c}-1) \mathrm{y}(1-\mathrm{y})\}=0 \\
& -2 a(1-c) y^{2}+(2-c(a b+1)) y+(a-1)=0 \\
& A y^{2}+B y+C=0 \\
& \mathrm{y}=\frac{-\mathrm{B} \pm \sqrt{\mathrm{B}^{2}-4 \mathrm{AC}}}{2 \mathrm{~A}}
\end{aligned}
$$


$\mathrm{x}=\left(\frac{-\mathrm{B} \pm \sqrt{\mathrm{B}^{2}-4 \mathrm{AC}}}{2 \mathrm{~A}}\right)^{1 / a}$

Where $y=x^{a}$

$A=-2 a(1-c)$

$B=2-c-a b c$

$C=(a-1)$

\subsection{Points of Inflection}

The points of the inflection of the LNK distribution are found from

$f^{\prime \prime}(x)=0$ under $f^{\prime \prime \prime}(x) \neq 0$ or equavalentally as

$\frac{d^{2} \ln f(x)}{d x^{2}}=0$ and $\frac{d^{3} \ln f(x)}{d x^{3}} \neq 0$

Where as

$$
\begin{aligned}
& \frac{d^{2} \ln f(x)}{d x^{2}}=\frac{-(a-1)}{x^{2}}-\frac{a(b-1) x^{a-2}\left[(a-1)+x^{a}\right]}{\left(1-\mathrm{x}^{a}\right)^{2}}+ \\
& \frac{a(1+b)(1-c) x^{a-2}\left[(a-1)+(1-c) x^{a}\right]}{\left[1-(1-c) x^{a}\right]^{2}}
\end{aligned}
$$

So

$$
\frac{-(a-1)}{x^{2}}-\frac{a(b-1) x^{a-2}\left[(a-1)+x^{a}\right]}{\left(1-\mathrm{x}^{a}\right)^{2}}+\frac{a(1+b)(1-c) x^{a-2}\left[(a-1)+(1-c) x^{a}\right]}{\left[1-(1-c) x^{a}\right]^{2}}=0
$$

After some Simplification

$$
A y^{4}+B y^{3}+C y^{2}+D y+E=0
$$

Where $y=x^{a}$

$$
\begin{aligned}
& A=-(a+1)(1-c)^{2} \\
& B=(1-c)\left[(2-c)\left(a+2-a^{2}\right)-a b c(1+a)\right] \\
& C=(1-c)\left(4 a^{2}-6\right)-a b c(c-2)-c^{2} \\
& D=(a-1)[(c-2)(a+2)+a b c] \\
& E=(a-1)
\end{aligned}
$$


Table 2.1 Points of inflection of LNK distribution

\begin{tabular}{|c|c|c|c|}
\hline A & B & C & Point (s) of inflection \\
\hline 1 & 2 & 0.1 & 0.8482 \\
\hline 1 & 2 & 0.2 & 0.6585 \\
\hline 1 & 2 & 0.3 & 0.0894 \\
\hline 1 & 2 & 0.4 & 0.7318 \\
\hline 1 & 3 & 0.1 & 0.3965 \\
\hline 1 & 3 & 0.2 & $0.4432,0.8678$ \\
\hline 2 & 3 & 0.1 & $0.5681,0.6607$ \\
\hline 2 & 3 & 0.2 & $0.4625,0.8089$ \\
\hline 2 & 4 & 0.1 & $0.4888,0.7454$ \\
\hline 2 & 5 & 0.1 & $0.5342,0.6644$ \\
\hline 2 & 6 & 0.1 & $0.5492,0.8741$ \\
\hline 3 & 4 & 0.1 & $0.5665,0.8335$ \\
\hline 3 & 5 & 0.1 & $0.5897,0.7881$ \\
\hline 3 & 6 & 0.1 & $0.6294,0.7273$ \\
\hline 3 & 7 & 0.1 & \\
\hline
\end{tabular}

\subsection{Bonferroni Curve}

The Bonferroni curve of the LNK distribution is as:

$$
\begin{aligned}
B_{F}(F(x)) & =\frac{1}{\mu F(x)} \int_{0}^{x} y f(y) d y \\
B_{F}(F(x)) & =\frac{\sum_{k=0}^{\infty} \frac{\Gamma\left(\frac{1}{a}+k\right)}{\Gamma\left(\frac{1}{a}\right) k !}\left(1-\frac{1}{c}\right)^{k} I\left(\frac{c x^{a}}{1-x^{a}+c x^{a}} ; \frac{1}{a}+k+1, b\right)}{\left(1-\left(\frac{1-x^{a}}{1-(1-c) x^{a}}\right)^{b}\right) \sum_{k=0}^{\infty} \frac{\Gamma\left(\frac{1}{a}+k\right)}{\Gamma\left(\frac{1}{a}\right) k !}\left(1-\frac{1}{c}\right)^{k} B\left(\frac{1}{a}+k+1, b\right)}
\end{aligned}
$$

\subsection{Lorenz Curve}

The Lorenz curve of the LNK distribution is as:

$$
L(F(x))=F(x) B(F(x))
$$




$$
L(F(x))=\frac{\sum_{k=0}^{\infty} \frac{\Gamma\left(\frac{1}{a}+k\right)}{\Gamma\left(\frac{1}{a}\right) k !}\left(1-\frac{1}{c}\right)^{k} I\left(\frac{c x^{a}}{1-x^{a}+c x^{a}} ; \frac{1}{a}+k+1, b\right)}{\sum_{k=0}^{\infty} \frac{\Gamma\left(\frac{1}{a}+k\right)}{\Gamma\left(\frac{1}{a}\right) k !}\left(1-\frac{1}{c}\right)^{k} B\left(\frac{1}{a}+k+1, b\right)}
$$

\subsection{Gini Coefficient}

The Gini coefficient of the LNK distribution is explained as:

$$
\begin{aligned}
G & =1-\mu^{-1} \int_{0}^{1}(1-F(x))^{2} d x \\
& =1-\frac{1}{\mu} \int_{0}^{1}\left(\frac{1-x^{a}}{1-(1-c) x^{a}}\right)^{2 b} d x
\end{aligned}
$$

\subsection{Mean deviations}

The mean deviation of the LNK distribution from arithmetic mean and median are denoted by $\delta_{1}(Y)$ and $\delta_{2}(Y)$ respectively and are found from

$$
\begin{aligned}
& \delta_{1}(Y)=\int_{0}^{1}|y-\mu| f(y) d y \\
& =\int_{0}^{\mu} \mu f(y) d y-\int_{0}^{\mu} y f(y) d y+\int_{\mu}^{1} y f(y) d y-\int_{\mu}^{1} \mu f(y) d y \\
& =2 \mu F(\mu)-2 J(\mu) \\
& =2 \mu\left(1-\left(\frac{1-\mu^{a}}{1-(1-c) \mu^{a}}\right)^{b}\right)-2 \frac{b}{c^{\frac{1}{a}}} \sum_{k=0}^{\infty} \frac{\Gamma\left(\frac{1}{a}+k\right)}{\Gamma\left(\frac{1}{a}\right) k !}\left(1-\frac{1}{c}\right)^{k} I\left(\frac{c \mu^{a}}{1-\mu^{a}+c \mu^{a}} ; \frac{1}{a}+k+1, b\right)
\end{aligned}
$$

The mean deviation about median

$$
\begin{aligned}
& \delta_{2}(Y)=\int_{0}^{1}|y-M| f(y) d y \\
& =\int_{0}^{M} M f(y) d y-\int_{0}^{M} y f(y) d y+\int_{M}^{1} y f(y) d y-\int_{M}^{1} M f(y) d y
\end{aligned}
$$




$$
\begin{aligned}
& =\mu-2 J(M) \\
& =\mu-2 \frac{b}{c^{\frac{1}{a}}} \sum_{k=0}^{\infty} \frac{\Gamma\left(\frac{1}{a}+k\right)}{\Gamma\left(\frac{1}{a}\right) k !}\left(1-\frac{1}{c}\right)^{k} I\left(\frac{c M^{a}}{1-M^{a}+c M^{a}} ; \frac{1}{a}+k+1, b\right)
\end{aligned}
$$

\section{ESTIMATION}

Here, we consider

\subsection{Maximum-likelihood estimation}

The likelihood function of random sample $x_{1}, x_{2}, \ldots, x_{n}$ of observation is given by

$$
\begin{aligned}
& f\left(x_{1}, x_{2}, \ldots, x_{n} ; \mathrm{a}, \mathrm{b}, \mathrm{c}\right)=\prod_{i=1}^{n} f\left(x_{i}, a, b, c\right) \\
& L\left(x_{i}, a, b, c\right)=\prod_{i=1}^{n} f\left(x_{i}, a, b, c\right) \\
& L\left(x_{i}, a, b, c\right)=\frac{(\mathrm{abc})^{n}\left(\prod_{i=1}^{n} x_{i}\right)^{a-1}\left(\prod_{i=1}^{n}\left(1-x_{i}^{a}\right)\right)^{b-1}}{\left(\prod_{i=1}^{n}\left(1-(1-c) x_{i}^{a}\right)\right)^{1+b}}
\end{aligned}
$$

Taking ln both sides

$\ln L=n \ln a b c+(a-1) \sum_{i=1}^{n} \ln x_{i}+(\mathrm{b}-1) \sum_{i=1}^{n} \ln \left(1-x_{i}^{a}\right)-(1+b) \sum_{i=1}^{n} \ln \left(1-(1-\mathrm{c}) x_{i}^{a}\right)$

It follows that the maximum-likelihood estimates $(\mathrm{a}, \mathrm{b}, \mathrm{c})$, say (迢 $\hat{\mathrm{b}}, \mathrm{c})$, are the simultaneous solutions of the equations:

$$
\begin{aligned}
& \frac{\partial L}{\partial a}=\frac{n}{a}+\sum_{i=1}^{n} \ln x_{i}-(b-1) \sum_{i=1}^{n} \frac{x_{i}^{a} \ln x_{i}}{\left(1-x_{i}^{a}\right)}+(1+b) \sum_{i=1}^{n} \frac{(1-c) x_{i}^{a} \ln x_{i}}{\left(1-(1-\mathrm{c}) \mathrm{x}_{i}{ }^{a}\right)} \\
& \frac{\partial L}{\partial b}=\frac{n}{b}+\sum_{i=1}^{n} \ln \left(1-x_{i}^{a}\right)-\sum_{i=1}^{n} \ln \left(1-(1-\mathrm{c}) \mathrm{x}_{i}{ }^{a}\right)
\end{aligned}
$$




$$
\frac{\partial L}{\partial c}=\frac{n}{c}-(1+b) \sum_{i=1}^{n} \frac{x_{i}^{a}}{1-(1-\mathrm{c}) \mathrm{x}_{i}^{a}}
$$

For the ML estimators, we solve the following three equations simultaneously we have

$$
\frac{\partial L}{\partial a}=0, \frac{\partial L}{\partial b}=0, \frac{\partial L}{\partial c}=0
$$

Since the equations are of implicit and have the complicated forms. Therefore, for numerically solution of the equations we can use differenent softwares like The R Mathematica 10.2 etc.

\section{SIMULATION STUDY}

Table 1 The Bias, MSE values for the LNK model when $a=b=c=2.0$

\begin{tabular}{|l|l|l|l|l|l|l|}
\hline & $\mathbf{n}=\mathbf{1 0}$ & $\mathbf{n}=\mathbf{2 0}$ & $\mathbf{n}=\mathbf{3 0}$ & $\mathbf{n}=\mathbf{5 0}$ & $\mathbf{n}=\mathbf{7 0}$ & $\mathbf{n}=\mathbf{1 0 0}$ \\
\hline Bias(a) & 0.6789 & 0.2966 & 0.1591 & 0.0732 & 0.0398 & 0.0134 \\
\hline Bias(b) & 148.4399 & 98.1754 & 80.9118 & 56.4929 & 42.0678 & 37.0448 \\
\hline Bias(c) & 519.4312 & 63.2427 & 5.1913 & 0.3873 & -0.1171 & -0.6091 \\
\hline MSE(a) & 2.3026 & 0.7537 & 0.3908 & 0.1723 & 0.1132 & 0.0843 \\
\hline MSE(b) & 45131.8214 & 22559.3813 & 17215.8524 & 9705.1161 & 5973.0134 & 5221.2081 \\
\hline MSE(c) & 4729852.1234 & 592121.2123 & 17854.2713 & 33.3845 & 15.21264 & 4.6655 \\
\hline & $\mathbf{n = 1 5 0}$ & $\mathbf{n}=\mathbf{2 0 0}$ & $\mathbf{n}=\mathbf{3 0 0}$ & $\mathbf{n}=500$ & $\mathbf{n}=\mathbf{7 0 0}$ & $\mathbf{n}=\mathbf{1 0 0 0}$ \\
\hline Bias(a) & 0.0165 & 0.0013 & 0.0025 & -0.0071 & -0.0157 & -0.0134 \\
\hline Bias(b) & 25.9659 & 17.9847 & 12.0603 & 6.9705 & 5.6428 & 4.7767 \\
\hline Bias(c) & -0.7730 & -0.9022 & -0.9606 & -0.9643 & -0.9604 & -0.9707 \\
\hline MSE(a) & 0.0578 & 0.0445 & 0.0286 & 0.0179 & 0.0123 & 0.0081 \\
\hline MSE(b) & 2688.8471 & 1429.0832 & 685.7248 & 176.8713 & 80.2722 & 36.4003 \\
\hline MSE(c) & 2.2229 & 1.8662 & 1.5139 & 1.3256 & 1.2007 & 1.1071 \\
\hline
\end{tabular}


Table 2 The Bias, MSE values for the LNK model when $a=b=c=2.5$

\begin{tabular}{|l|l|l|l|l|l|l|}
\hline & $\mathbf{n}=\mathbf{1 0}$ & $\mathbf{n}=\mathbf{2 0}$ & $\mathbf{n}=\mathbf{3 0}$ & $\mathbf{n}=\mathbf{5 0}$ & $\mathbf{n}=\mathbf{7 0}$ & $\mathbf{n}=\mathbf{1 0 0}$ \\
\hline Bias(a) & 0.7101 & 0.1423 & 0.0226 & -0.0336 & -0.0764 & -0.0892 \\
\hline Bias(b) & 148.6944 & 83.5053 & 70.3768 & 49.9135 & 40.9383 & 28.8218 \\
\hline Bias(c) & 908.6814 & 240.0717 & 101.7437 & 6.0567 & 3.3570 & 1.6298 \\
\hline MSE(a) & 2.5889 & 0.6457 & 0.4488 & 0.2476 & 0.1627 & 0.1103 \\
\hline MSE(b) & 46025.2324 & 19184.2612 & 13459.1232 & 7983.5764 & 5993.6893 & 3566.5871 \\
\hline MSE(c) & 8932954 & 2172753 & 858508 & 1049.3921 & 181.7757 & 45.5042 \\
\hline & $\mathbf{n = 1 5 0}$ & $\mathbf{n}=\mathbf{2 0 0}$ & $\mathbf{n}=\mathbf{3 0 0}$ & $\mathbf{n}=500$ & $\mathbf{n}=\mathbf{7 0 0}$ & $\mathbf{n}=\mathbf{1 0 0 0}$ \\
\hline Bias(a) & -0.1165 & -0.1040 & -0.1170 & -0.1267 & -0.1286 & -0.1309 \\
\hline Bias(b) & 16.4083 & 11.5937 & 8.2968 & 4.7545 & 3.6887 & 3.1612 \\
\hline Bias(c) & 0.9505 & 1.0242 & 0.6185 & 0.4136 & 0.4096 & 0.2923 \\
\hline MSE(a) & 0.0804 & 0.0619 & 0.0491 & 0.0373 & 0.0310 & 0.0282 \\
\hline MSE(b) & 1369.2381 & 791.9238 & 402.1093 & 108.3226 & 41.8772 & 16.9204 \\
\hline MSE(c) & 15.8173 & 13.1791 & 6.4383 & 3.0282 & 2.2463 & 1.4012 \\
\hline
\end{tabular}

Table 3 The Bias, MSE values for the LNK model when $a=b=c=3.0$

\begin{tabular}{|l|l|l|l|l|l|l|}
\hline & $\mathbf{n}=\mathbf{1 0}$ & $\mathbf{n}=\mathbf{2 0}$ & $\mathbf{n}=\mathbf{3 0}$ & $\mathbf{n}=\mathbf{5 0}$ & $\mathbf{n}=\mathbf{7 0}$ & $\mathbf{n}=\mathbf{1 0 0}$ \\
\hline Bias(a) & 0.4603 & 0.0535 & -0.0688 & -0.1884 & -0.2438 & -0.2725 \\
\hline Bias(b) & 139.5197 & 81.7559 & 59.6459 & 39.1355 & 28.2413 & 16.5283 \\
\hline Bias(c) & 1318.3864 & 540.5148 & 167.8884 & 61.5224 & 12.9443 & 9.0277 \\
\hline MSE(a) & 2.1234 & 0.9155 & 0.6307 & 0.3236 & 0.2394 & 0.2077 \\
\hline MSE(b) & 43208.6434 & 18732.8834 & 11124.5823 & 5996.8751 & 3686.9514 & 2041.9723 \\
\hline MSE(c) & 12800727 & 4713924 & 1058158 & 285476.4 & 1760.2651 & 455.9306 \\
\hline & $\mathbf{n = 1 5 0}$ & $\mathbf{n}=\mathbf{2 0 0}$ & $\mathbf{n}=\mathbf{3 0 0}$ & $\mathbf{n}=500$ & $\mathbf{n}=\mathbf{7 0 0}$ & $\mathbf{n}=\mathbf{1 0 0 0}$ \\
\hline $\operatorname{Bias(a)}$ & -0.2895 & -0.3107 & -0.3037 & -0.3063 & -0.3198 & -0.3133 \\
\hline Bias(b) & 10.2456 & 6.4328 & 3.8052 & 2.2502 & 1.5573 & 1.3494 \\
\hline $\operatorname{Bias(c)}$ & 7.5233 & 7.4066 & 5.9516 & 5.0419 & 5.2533 & 4.9514 \\
\hline MSE(a) & 0.1698 & 0.1596 & 0.1384 & 0.1194 & 0.1204 & 0.1101 \\
\hline MSE(b) & 845.9625 & 368.9467 & 139.7221 & 35.0732 & 8.4393 & 3.1515 \\
\hline MSE(c) & 238.0884 & 154.0213 & 83.8912 & 46.6563 & 42.8393 & 34.4719 \\
\hline
\end{tabular}




\section{SOME TRANSFORMED DISTRIBUTIONS}

5.1 Following table show the dffierent transformation of LNK distribution:

\begin{tabular}{|c|c|c|c|c|c|}
\hline Sr. No. & $\mathbf{a}$ & b & c & Transformation & Resulting Distribution \\
\hline 1 & $a$ & $b$ & 1 & $X$ & Kumaraswamy distribution (1980) \\
\hline 2 & $a$ & $b$ & 1 & $1-x$ & $\begin{array}{l}\text { Corderio (2012) distribution } \\
F(x)=1-\left(1-(1-x)^{a}\right)^{b} \\
0<x<1, \text { a }, \mathrm{b}>0\end{array}$ \\
\hline 3 & 1 & $b$ & 1 & $X$ & $\begin{array}{l}\mathrm{B}(1, \mathrm{~b}) \text { and } \operatorname{Kum}(1, \mathrm{~b}) \\
\text { Power distribution }\end{array}$ \\
\hline 4 & $a$ & 1 & 1 & $X$ & $\begin{array}{l}\mathrm{B}(\mathrm{a}, 1) \text { and } \operatorname{Kum}(\mathrm{a}, 1) \\
\text { Power distribution }\end{array}$ \\
\hline 5 & $a$ & 1 & 1 & $-\log x$ & $\operatorname{Exp}(\mathrm{a})$ \\
\hline 6 & 1 & $b$ & 1 & $-\log (1-x)$ & $\operatorname{Exp}(b)$ \\
\hline 7 & $a$ & $b$ & 1 & $X$ & $\operatorname{Kum}(\mathrm{a}, \mathrm{b})$ or $\mathrm{G} B_{1}(\mathrm{a}, 1,1, \mathrm{~b})$ \\
\hline 8 & 1 & $b$ & 1 & $\frac{x}{1+x}$ & $\begin{array}{l}F(x)=1-\frac{1}{(1+x)^{b}} \\
0<x<\infty, \quad b>0\end{array}$ \\
\hline 9 & 1 & $b$ & C & $\frac{c x}{1-x+c x}$ & $\begin{array}{l}F(x)=1-(1-x)^{b} \\
0<x<1, \quad b>0\end{array}$ \\
\hline 10 & 1 & 1 & 1 & $X$ & Uniform $(0,1)$ \\
\hline
\end{tabular}

\subsection{Asymptotes and Shapes}

The asymptotes of (1.1), (1.2) and (3.1) as $x \rightarrow 0,1$ are given by

\begin{tabular}{|c|c|c|}
\hline & $p d f(x)$ & hrf $h(x)$ \\
\hline$x \rightarrow 0$ & $a b c x^{\mathrm{a}-1}$ where $a, b, c<1$ & $a b c x^{\mathrm{a}-1}$ where $a, b, c<1$ \\
\hline \multirow{2}{*}{$x \rightarrow 1$} & $a b c\left(1-x^{a}\right)^{\mathrm{b}-1}$ where $a, b, c<1$ & $\frac{a b c}{\left(1-x^{a}\right)}$ where $a, b, c<1$ \\
\hline
\end{tabular}




\section{APPLICATION}

In order to prove that LNK distribution can be a better model than the Power distribution, Beta distribution with $(\mathrm{a}=1)$, Beta distribution, Kumaraswamy distribution, let us use three real data sets.

The following tables show the numerical values with MLEs and their corresponding standard errors (in parentheses) of the model parameters including loglikelihood, KolmogorovSmirnov test (KS), Akaike information criterion (AIC) and Consistent Akaike information criterion (CAIC) for comparing LNK distribution with the Power distribution, Beta distribution with $(\mathrm{a}=1)$, Beta distribution, Kumaraswamy distribution. It is quite evident from the reports that LNK distribution is better. The plots of the fitted distributions to real datasets are shown in figures.

\section{Data Set 1:}

The following right to skewed dataset presented by Cordeiro and Brito (2012) [13] is obtained from the measurements on petroleum rock samples. The data consists of 48 rock samples from a petroleum reservoir. The dataset corresponds to twelve core samples from petroleum reservoirs that were sampled by four cross-sections. Each core sample was measured for permeability and each cross-section has the following variables: the total area of pores, the total perimeter of pores and shape. We analyze the shape perimeter by squared (area) variable and the observations are:

$\begin{array}{llllll}\text { 0.0903296, } & 0.2036540, & 0.2043140, & 0.2808870, & 0.1976530, & 0.3286410, \\ \text { 0.1486220, } & 0.1623940, & 0.2627270, & 0.1794550, & 0.3266350, & 0.2300810, \\ \text { 0.1833120, } & 0.1509440, & 0.2000710, & 0.1918020, & 0.1541920, & 0.4641250, \\ 0.1170630, & 0.1481410, & 0.1448100, & 0.1330830, & 0.2760160, & 0.4204770, \\ 0.1224170, & 0.2285950, & 0.1138520, & 0.2252140, & 0.1769690, & 0.2007440, \\ 0.1670450, & 0.2316230, & 0.2910290, & 0.3412730, & 0.4387120, & 0.2626510, \\ 0.1896510, & 0.1725670, & 0.2400770, & 0.3116460, & 0.1635860, & 0.1824530, \\ 0.1641270, & 0.1534810, & 0.1618650, & 0.2760160, & 0.2538320, & 0.2004470 .\end{array}$

\section{Data set 2}

The symmetric behavior of the following dataset, discussed by Dasgupta (2011) [14], consists of 50 observations relates to holes operation on jobs made of iron sheet. This dataset is as follows: $0.04,0.02,0.06,0.12,0.14,0.08,0.22,0.12,0.08,0.26,0.24,0.04,0.14,0.16,0.08,0.26,0.32,0.28,0.14$, $0.16,0.24,0.22,0.12,0.18,0.24,0.32,0.16,0.14,0.08,0.16,0.24,0.16,0.32,0.18,0.24,0.22,0.16,0.12$, $0.24,0.06,0.02,0.18,0.22,0.14,0.06,0.04,0.14,0.26,0.18,0.16$. 


\section{Data Set 3:}

The following second data set presented by Cordeiro and Brito (2012) [13], displays the skewed symmetric trend of data. This data discusses the total milk production in the first birth of 107 cows from SINDI race. These cows are property of the Carnaúba farm which belongs to the Agropecuária Manoel Dantas Ltda (AMDA), located in Taperoá City, Paraíba (Brazil). The data of proportion of total milk is as under:

0.4365, 0.4260, 0.5140, 0.6907, 0.7471, 0.2605, 0.6196, 0.8781, 0.4990, 0.6058, 0.6891, 0.5770, 0.5394, $0.1479,0.2356,0.6012,0.1525,0.5483,0.6927,0.7261,0.3323,0.0671,0.2361,0.4800,0.5707,0.7131$, $0.5853,0.6768,0.5350,0.4151,0.6789,0.4576,0.3259,0.2303,0.7687,0.4371,0.3383,0.6114,0.3480$, $0.4564,0.7804,0.3406,0.4823,0.5912,0.5744,0.5481,0.1131,0.7290,0.0168,0.5529,0.4530,0.3891$, 0.4752, 0.3134, 0.3175,0.1167, 0.6750, 0.5113, 0.5447, 0.4143,0.5627, 0.5150, 0.0776, 0.3945, 0.4553, $0.4470,0.5285,0.5232,0.6465,0.0650,0.8492,0.8147,0.3627,0.3906,0.4438,0.4612,0.3188,0.2160$, 0.6707, 0.6220, 0.5629, 0.4675, 0.6844, 0.3413, 0.4332, 0.0854, 0.3821, 0.4694, 0.3635, 0.4111, 0.5349, 0.3751, 0.1546, 0.4517, 0.2681, 0.4049, 0.5553, 0.5878, 0.4741, 0.3598, 0.7629, 0.5941, 0.6174, 0.6860, 0.0609, 0.6488, 0.2747.

Estimated Parameters by MLE with their S.E. and Goodness of Fit

Data set 1

\begin{tabular}{|c|c|c|c|c|c|c|c|}
\hline Model & $\hat{\alpha}$ & $\hat{\beta}$ & $\hat{\gamma}$ & $-\ln (L)$ & KS & AIC & CAIC \\
\hline Power & $\begin{array}{l}0.63 \\
(0.0909)\end{array}$ & & & -6.0118 & 0.4295 & 14.0237 & 14.1107 \\
\hline $\begin{array}{l}\text { Beta } \\
(\alpha=1)\end{array}$ & & $\begin{array}{l}3.9643 \\
(0.5722)\end{array}$ & & $\begin{array}{l}- \\
30.2205\end{array}$ & 0.9156 & 62.4412 & 62.5281 \\
\hline Kumaraswamy & $\begin{array}{l}44.6597 \\
(17.5894)\end{array}$ & $\begin{array}{l}2.7187 \\
(0.2937)\end{array}$ & & $\begin{array}{l}- \\
52.4915\end{array}$ & 0.1533 & 108.9831 & 109.2497 \\
\hline Beta & $\begin{array}{l}5.9417 \\
(1.1825)\end{array}$ & $\begin{array}{l}21.2057 \\
(4.3513)\end{array}$ & & $\begin{array}{l}- \\
55.6002\end{array}$ & 0.1427 & 115.2004 & 115.4671 \\
\hline $\begin{array}{l}\text { Libby Novick } \\
\text { Kumaraswamy }\end{array}$ & $\begin{array}{l}5.5040 \\
(0.6078)\end{array}$ & $\begin{array}{l}0.7469 \\
(0.2042)\end{array}$ & $\begin{array}{l}10.4007 \\
(130.7012)\end{array}$ & $\begin{array}{l}- \\
57.7939\end{array}$ & 0.0852 & 121.588 & 122.1334 \\
\hline
\end{tabular}




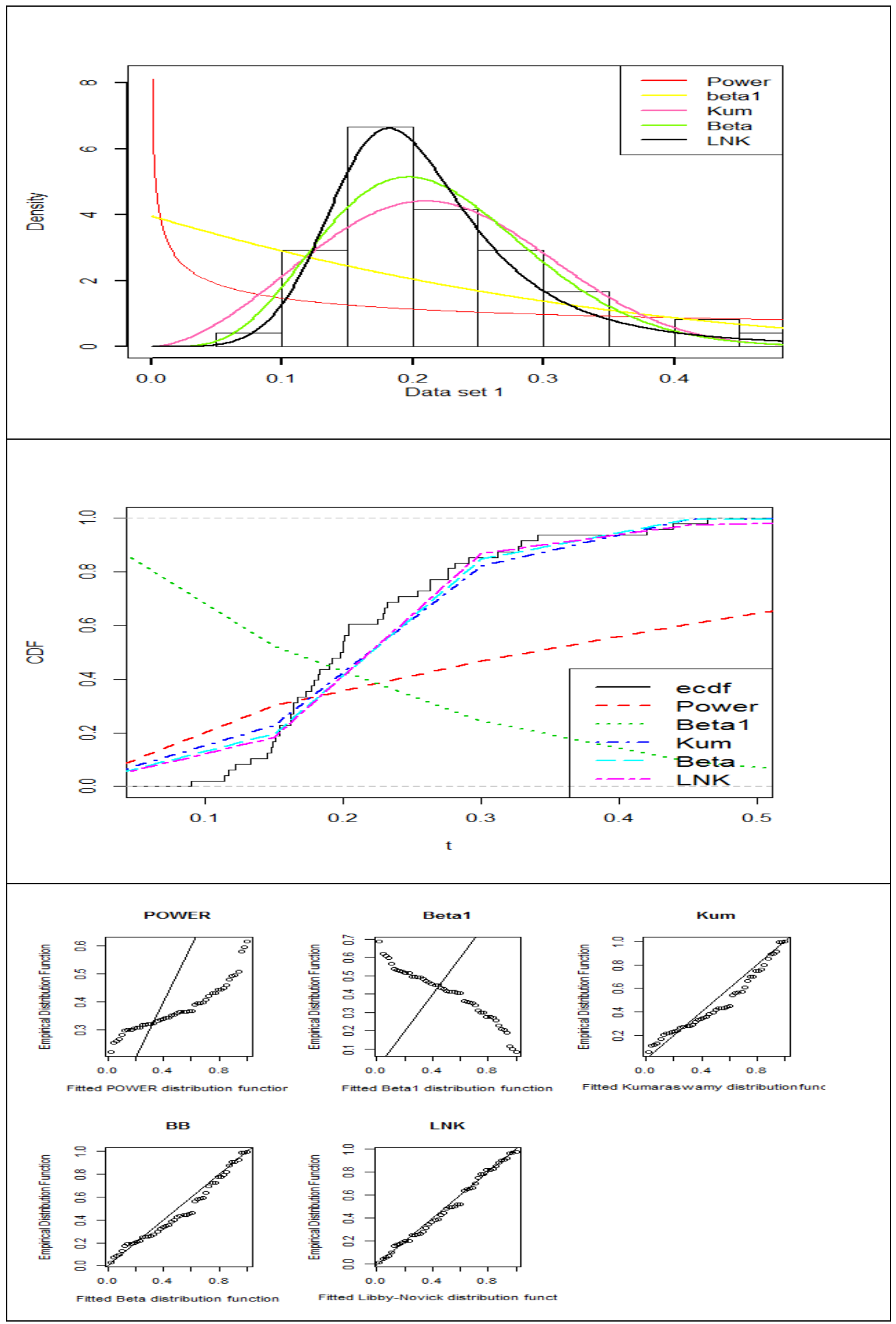

Figure 6.1: $p d f$, $c d f$ and $Q-Q$ graphs of the densities for data set 1 
Estimated Parameters by MLE with their S.E. and Goodness of Fit

Data set 2

\begin{tabular}{|l|l|l|l|l|l|l|l|}
\hline Model & $\hat{\alpha}$ & $\hat{\beta}$ & $\hat{\gamma}$ & $-\ln (L)$ & KS & AIC & CAIC \\
\hline Power & $\begin{array}{l}0.5033 \\
(0.0711)\end{array}$ & & & -15.0139 & 0.4364 & 32.0279 & 32.1113 \\
\hline $\begin{array}{l}\text { Beta } \\
(\alpha=1)\end{array}$ & & $\begin{array}{l}5.4693 \\
(0.7735)\end{array}$ & & -44.0998 & 0.8786 & 90.1997 & 90.2831 \\
\hline Kumaraswamy & $\begin{array}{l}33.1367 \\
(13.9342)\end{array}$ & $\begin{array}{l}2.0773 \\
(0.2551)\end{array}$ & & -56.0686 & 0.0902 & 116.1374 & 116.3927 \\
\hline Beta & $\begin{array}{l}2.6825 \\
(0.5074)\end{array}$ & $\begin{array}{l}13.8656 \\
(2.8295)\end{array}$ & & -54.6066 & 0.1214 & 113.2133 & 113.4686 \\
\hline Kibby Novick & 2.0376 & 226.0664 & 0.1342 & -56.2244 & 0.0900 & 118.4489 & 118.9707 \\
$(0.2687)$ & $(980.0583)$ & $(0.5945)$ & & & & \\
\hline
\end{tabular}

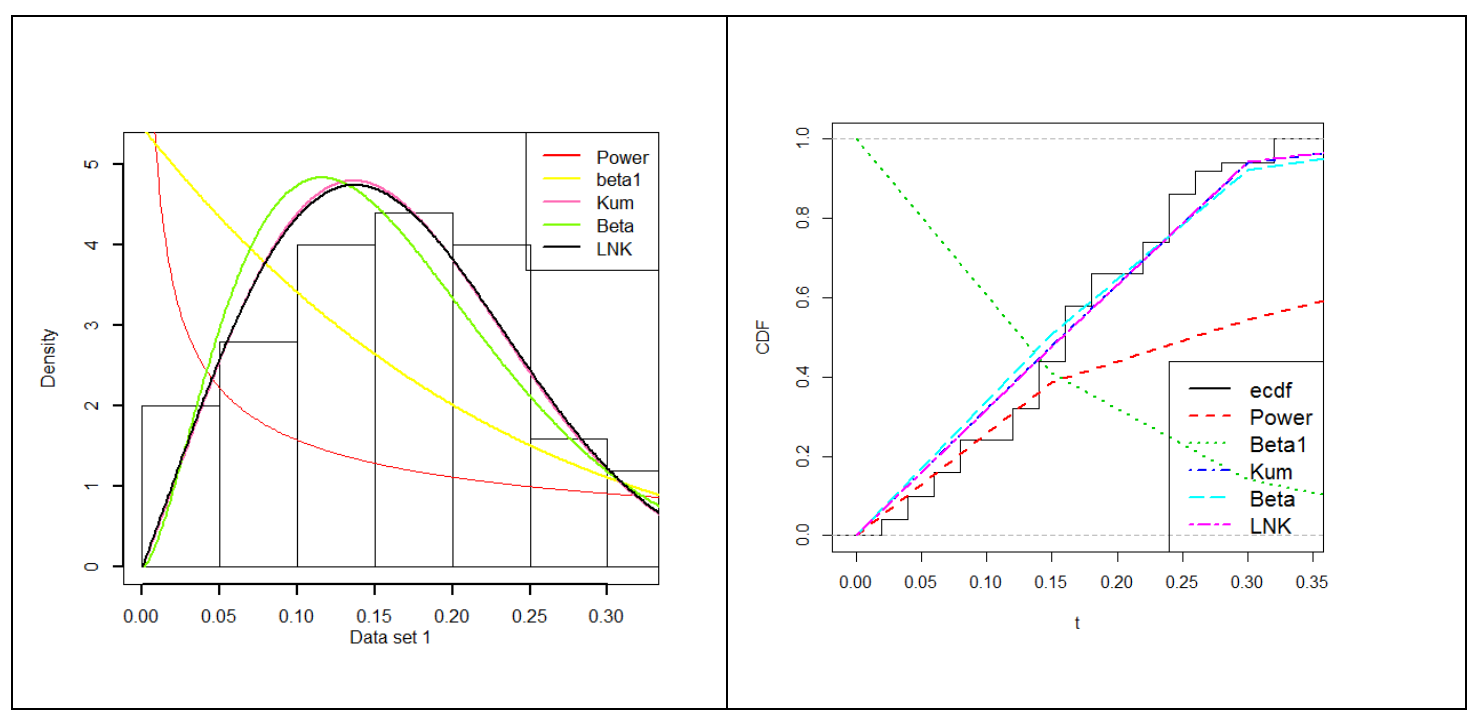

Figure 6.2: $p d f$ and $c d f$ graphs of the compitators distributions for data set 2 

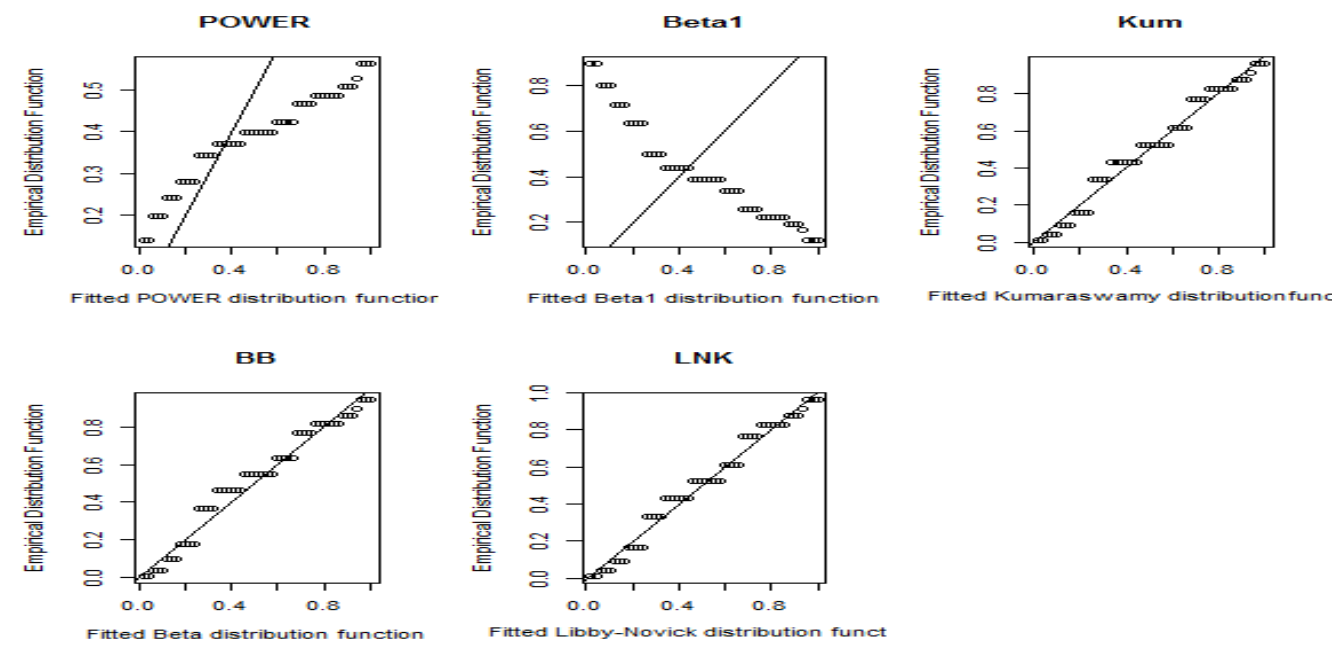

Figure 6.3: $Q-Q$ plots of the densities for data set 2

Estimated Parameters by MLE with their S.E. and Goodness of Fit data3

\begin{tabular}{|l|l|l|l|l|l|l|l|}
\hline Model & $\hat{\alpha}$ & $\hat{\beta}$ & $\hat{\gamma}$ & $-\ln (L)$ & KS & AIC & CAIC \\
\hline Power & $\begin{array}{l}1.1123 \\
(0.1075)\end{array}$ & & & -0.58545 & 0.2418 & 3.17090 & 3.209 \\
$(\alpha=1)$ & & $\begin{array}{l}1.4181 \\
(0.1371)\end{array}$ & & -5.8304 & 0.9669 & 13.6608 & 13.6989 \\
\hline Kumaraswamy & 3.4363 & 2.1948 & & -25.3946 & 0.0769 & 54.7893 & 54.9047 \\
& $(0.5822)$ & $(0.2224)$ & & & & & \\
\hline Beta & 2.4125 & 2.8296 & & -23.7772 & 0.0816 & 51.5544 & 51.6698 \\
& $(0.3145)$ & $(0.3745)$ & & & & & \\
\hline Libby Novick Kumaraswamy & 1.7588 & 18.3624 & 0.1044 & -27.2446 & 0.0698 & 60.4892 & 60.7222 \\
& $(1.0411)$ & $(201.0970)$ & $(1.2976)$ & & & & \\
\hline
\end{tabular}



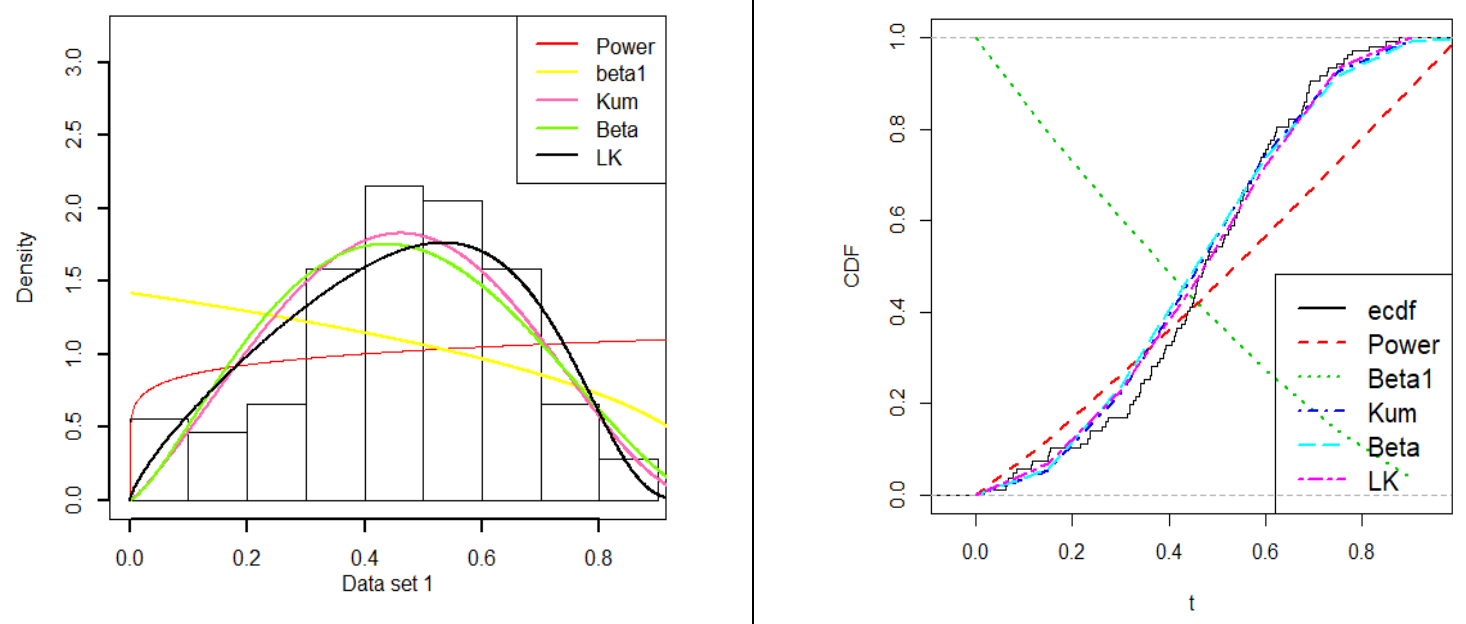

Figure 6.4: $p d f$ and $c d f$ graphs of the compitators distributions for data set 3

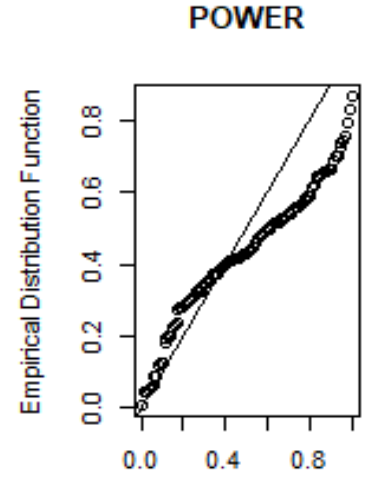

Fitted POWER distribution functio

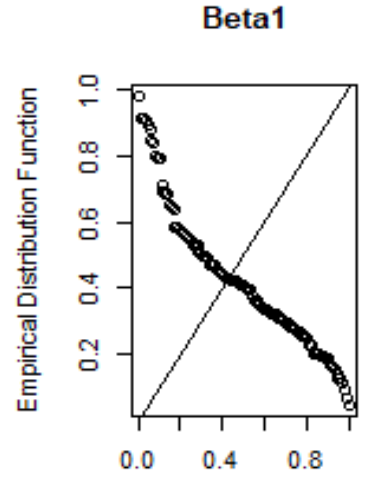

Fitted Beta1 distribution functior

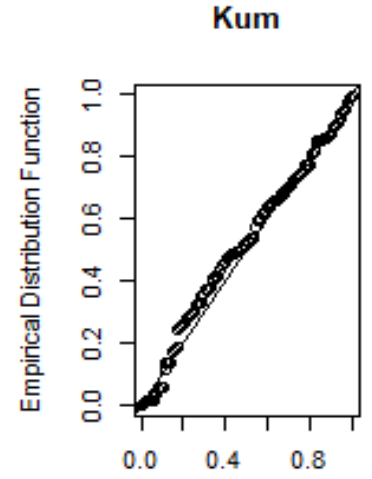

Fitted Kumaraswamy distributionfur
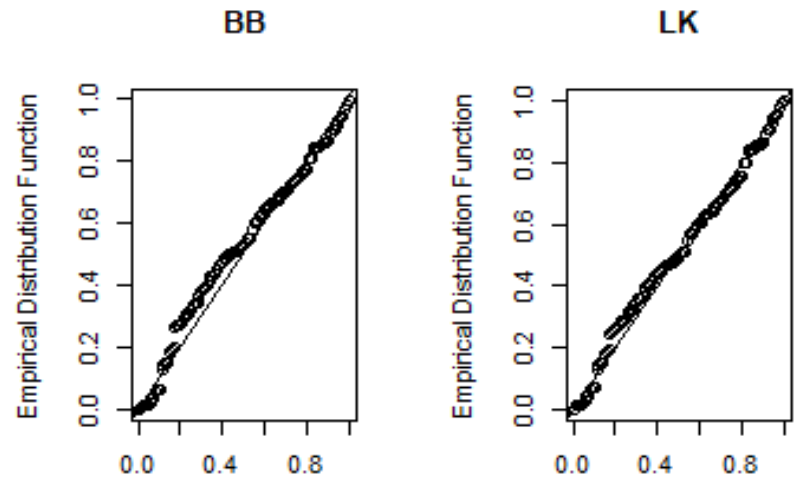

Fitted Beta distribution function

Fitted Libby-Novick distribution func

Figure 6.5: $Q-Q$ plots of the densities for data set 3 


\section{CONCLUSION}

The LNK distribution has some properties like the LNB distribution but, there are numerous benefits of the LNK distribution over LNB distribution: the LNK distribution has simple closed form of both its $c d f$ and quantile function and that's why it is easy to use in simulation studies. The distribution and quantile function of LNK do not involve any special functions. To compare the proposed model with other models, we apply these models to three sets of real data from different fields of science and engineering and it is examined by using well-known statistics. We conclude that the LNK distribution is better than the power, Beta with $(a=1)$, Beta distribution and Kumaraswamy distribution. 


\section{Appendix 1: Quantile values of LNK distribution}

\begin{tabular}{|c|c|c|c|c|c|c|c|c|c|c|c|c|c|}
\hline $\mathrm{a}$ & $\mathrm{b}$ & $\mathrm{c}$ & 0.1 & 0.2 & 0.3 & 0.4 & 0.5 & 0.6 & 0.7 & 0.8 & 0.9 & 0.99 & 0.999 \\
\hline 0.5 & 0.5 & 0.1 & 0.49155 & 0.72089 & 0.83237 & 0.89632 & 0.93652 & 0.96296 & 0.98050 & 0.99171 & 0.99798 & 0.99998 & 1 \\
\hline 0.5 & 0.5 & 0.2 & 0.29135 & 0.54420 & 0.70361 & 0.80797 & 0.87890 & 0.92795 & 0.96158 & 0.98353 & 0.99597 & 0.99996 & 1 \\
\hline 0.5 & 0.5 & 0.6 & 0.07899 & 0.23413 & 0.40237 & 0.55900 & 0.69444 & 0.80539 & 0.89110 & 0.95181 & 0.98798 & 0.99988 & 0.99999 \\
\hline 0.5 & 0.5 & 1.0 & 0.03610 & 0.12960 & 0.26010 & 0.40960 & 0.56250 & 0.70560 & 0.82810 & 0.92160 & 0.98010 & 0.99980 & 0.99999 \\
\hline 0.5 & 0.5 & 1.5 & 0.01828 & 0.07438 & 0.16780 & 0.29416 & 0.44444 & 0.60493 & 0.75831 & 0.88581 & 0.97037 & 0.99970 & 0.99999 \\
\hline 0.5 & 0.5 & 3.0 & 0.00525 & 0.02493 & 0.06634 & 0.13845 & 0.25000 & 0.40495 & 0.59472 & 0.79012 & 0.94204 & 0.99940 & 0.99999 \\
\hline 0.5 & 0.5 & 6.0 & 0.00141 & 0.00734 & 0.02185 & 0.05224 & 0.11111 & 0.21777 & 0.39386 & 0.64000 & 0.88898 & 0.99880 & 0.99998 \\
\hline 0.5 & 0.5 & 10 & 0.00052 & 0.00283 & 0.00888 & 0.02278 & 0.05325 & 0.11851 & 0.25277 & 0.49827 & 0.82493 & 0.99800 & 0.99998 \\
\hline 1.0 & 1.0 & 0.1 & 0.52631 & 0.71428 & 0.81081 & 0.86956 & 0.90909 & 0.93750 & 0.95890 & 0.97561 & 0.98901 & 0.99899 & 0.99990 \\
\hline 1.0 & 1.0 & 0.2 & 0.35714 & 0.55555 & 0.68181 & 0.76923 & 0.83333 & 0.88235 & 0.92105 & 0.95238 & 0.97826 & 0.99798 & 0.99980 \\
\hline 1.0 & 1.0 & 0.6 & 0.15625 & 0.29411 & 0.41666 & 0.52631 & 0.62500 & 0.71428 & 0.79545 & 0.86956 & 0.93750 & 0.99397 & 0.99940 \\
\hline 1.0 & 1.0 & 1.0 & 0.10000 & 0.20000 & 0.30000 & 0.40000 & 0.50000 & 0.60000 & 0.70000 & 0.80000 & 0.90000 & 0.99000 & 0.99900 \\
\hline 1.0 & 1.0 & 1.5 & 0.06896 & 0.14285 & 0.22222 & 0.30769 & 0.40000 & 0.50000 & 0.60869 & 0.72727 & 0.85714 & 0.98507 & 0.99850 \\
\hline 1.0 & 1.0 & 3.0 & 0.03571 & 0.07692 & 0.12500 & 0.18181 & 0.25000 & 0.33333 & 0.43750 & 0.57142 & 0.75000 & 0.97058 & 0.99700 \\
\hline 1.0 & 1.0 & 6.0 & 0.01818 & 0.04000 & 0.06666 & 0.10000 & 0.14285 & 0.20000 & 0.28000 & 0.40000 & 0.60000 & 0.94285 & 0.99403 \\
\hline 1.0 & 1.0 & 10.0 & 0.01098 & 0.02439 & 0.04109 & 0.06250 & 0.0909 & 0.13043 & 0.18918 & 0.28571 & 0.47368 & 0.90825 & 0.99008 \\
\hline 2.0 & 2.0 & 0.1 & 0.59248 & 0.73576 & 0.81319 & 0.86269 & 0.89751 & 0.92368 & 0.94444 & 0.96184 & 0.97764 & 0.99449 & 0.998371 \\
\hline 2.0 & 2.0 & 0.2 & 0.46139 & 0.60921 & 0.70282 & 0.76984 & 0.82120 & 0.86253 & 0.89722 & 0.92775 & 0.95673 & 0.98907 & 0.99675 \\
\hline 2.0 & 2.0 & 0.6 & 0.28757 & 0.40544 & 0.49548 & 0.57148 & 0.63906 & 0.70143 & 0.76103 & 0.82049 & 0.88475 & 0.96824 & 0.99034 \\
\hline
\end{tabular}




\begin{tabular}{|l|l|l|l|l|l|l|l|l|l|l|l|l|l|}
\hline 2.0 & 2.0 & 1.0 & 0.22653 & 0.32492 & 0.40415 & 0.47476 & 0.54119 & 0.60625 & 0.67251 & 0.74349 & 0.82690 & 0.94868 & 0.98406 \\
\hline 2.0 & 2.0 & 1.5 & 0.18656 & 0.27009 & 0.33935 & 0.40308 & 0.46517 & 0.52843 & 0.59585 & 0.67213 & 0.76838 & 0.92582 & 0.97637 \\
\hline 2.0 & 2.0 & 3.0 & 0.13308 & 0.19456 & 0.24718 & 0.29735 & 0.34831 & 0.40283 & 0.46458 & 0.54018 & 0.64719 & 0.86602 & 0.95434 \\
\hline 2.0 & 2.0 & 6.0 & 0.09452 & 0.13889 & 0.17751 & 0.21507 & 0.25412 & 0.29715 & 0.34781 & 0.41330 & 0.51469 & 0.77459 & 0.91442 \\
\hline 2.0 & 2.0 & 10.0 & 0.07334 & 0.10800 & 0.13838 & 0.16815 & 0.19943 & 0.23435 & 0.27618 & 0.33167 & 0.42164 & 0.68824 & 0.86823 \\
\hline 2.5 & 2.5 & 1.0 & 0.27941 & 0.37373 & 0.44615 & 0.50896 & 0.56705 & 0.62340 & 0.68063 & 0.74227 & 0.81622 & 0.93330 & 0.97426 \\
\hline 3.0 & 3.0 & 0.1 & 0.64095 & 0.75811 & 0.82327 & 0.86619 & 0.89717 & 0.9210 & 0.94038 & 0.95702 & 0.97268 & 0.99101 & 0.99632 \\
\hline 3.0 & 3.0 & 0.2 & 0.53323 & 0.65307 & 0.72871 & 0.78371 & 0.82677 & 0.86225 & 0.89283 & 0.92060 & 0.94813 & 0.98233 & 0.99270 \\
\hline 3.0 & 3.0 & 0.6 & 0.38309 & 0.48491 & 0.55810 & 0.61822 & 0.67111 & 0.71995 & 0.76713 & 0.81531 & 0.86978 & 0.95043 & 0.97871 \\
\hline 3.0 & 3.0 & 1.0 & 0.32557 & 0.41540 & 0.48216 & 0.53897 & 0.59088 & 0.64085 & 0.69143 & 0.74602 & 0.81222 & 0.92230 & 0.965489 \\
\hline 3.0 & 3.0 & 1.5 & 0.28551 & 0.36582 & 0.42659 & 0.47932 & 0.52858 & 0.57723 & 0.62799 & 0.68488 & 0.75764 & 0.89138 & 0.94991 \\
\hline 3.0 & 3.0 & 3.0 & 0.22749 & 0.29276 & 0.34308 & 0.38769 & 0.43040 & 0.47385 & 0.52089 & 0.57626 & 0.65255 & 0.81847 & 0.90856 \\
\hline 3.0 & 3.0 & 6.0 & 0.18092 & 0.23334 & 0.27416 & 0.31075 & 0.34627 & 0.38301 & 0.42366 & 0.47297 & 0.54441 & 0.72284 & 0.84343 \\
\hline 3.0 & 3.0 & 10.0 & 0.15271 & 0.19714 & 0.23187 & 0.26316 & 0.29369 & 0.32550 & 0.36102 & 0.40471 & 0.46950 & 0.64388 & 0.77952 \\
\hline
\end{tabular}




\section{Appendix 2: Variance, Skewness and Kurtosis of LNK distribution}

Following table shows the skewness and kutosis alongwith variance of LNK distribution

\begin{tabular}{|c|c|c|c|c|c|}
\hline $\mathrm{a}$ & $\mathrm{b}$ & $\mathrm{c}$ & Variance & Skewness & Kurtosis \\
\hline 0.5 & 0.5 & 0.1 & 0.05296 & -1.90608 & 6.01753 \\
\hline 0.5 & 0.5 & 0.2 & 0.07621 & -1.27182 & 3.53694 \\
\hline 0.5 & 0.5 & 0.6 & 0.11146 & -0.46476 & 1.80245 \\
\hline 0.5 & 0.5 & 1 & 0.12190 & -0.13530 & 1.5386 \\
\hline 0.5 & 0.5 & 1.5 & 0.12589 & 0.11638 & 1.50603 \\
\hline 0.5 & 0.5 & 3.0 & 0.12346 & 0.54039 & 1.77633 \\
\hline 0.5 & 0.5 & 6.0 & 0.11145 & 0.97311 & 2.46619 \\
\hline 0.5 & 0.5 & 10.0 & 0.09890 & 1.30763 & 3.28243 \\
\hline 1.0 & 1.0 & 0.1 & 0.04264 & -1.82849 & 5.97880 \\
\hline 1.0 & 1.0 & 0.2 & 0.05954 & -1.19142 & 3.56560 \\
\hline 1.0 & 1.0 & 0.6 & 0.08049 & -0.35626 & 1.95722 \\
\hline 1.0 & 1.0 & 1 & 0.08333 & 0.00000 & 1.80000 \\
\hline 1.0 & 1.0 & 1.5 & 0.08152 & 0.28209 & 1.89855 \\
\hline 1.0 & 1.0 & 3.0 & 0.07109 & 0.78491 & 2.56442 \\
\hline 1.0 & 1.0 & 6.0 & 0.05508 & 1.34776 & 4.06188 \\
\hline 1.0 & 1.0 & 10.0 & 0.04264 & 1.82849 & 5.97880 \\
\hline 1.5 & 1.5 & 0.1 & 0.03501 & -87.1461 & 386.365 \\
\hline 1.5 & 1.5 & 0.2 & 0.04780 & -40.6365 & 139.708 \\
\hline 1.5 & 1.5 & 0.6 & 0.06162 & -13.6714 & 32.6900 \\
\hline 1.5 & 1.5 & 1 & 0.06205 & -0.04546 & 2.00878 \\
\hline 1.5 & 1.5 & 1.5 & 0.05922 & 0.21997 & 2.1023 \\
\hline 1.5 & 1.5 & 3.0 & 0.04919 & 0.68276 & 2.73117 \\
\hline 1.5 & 1.5 & 6.0 & 0.03603 & 1.18148 & 4.11004 \\
\hline 1.5 & 1.5 & 10.0 & 0.02662 & 1.58988 & 5.82322 \\
\hline 2.0 & 2.0 & 0.1 & 0.02935 & -1.80078 & 6.22199 \\
\hline 2.0 & 2.0 & 0.2 & 0.03939 & -1.20368 & 3.91541 \\
\hline 2.0 & 2.0 & 0.6 & 0.04923 & -0.43784 & 2.35643 \\
\hline 2.0 & 2.0 & 1 & 0.04888 & -0.12530 & 2.18005 \\
\hline 2.0 & 2.0 & 3.0 & 0.03789 & 0.50497 & 2.69308 \\
\hline 2.0 & 2.0 & 6.0 & 0.02764 & 0.89112 & 3.64976 \\
\hline 2.0 & 2.0 & 10.0 & 0.02052 & 1.17422 & 4.71545 \\
\hline 2.5 & 2.5 & 0.1 & 0.02502 & -694.098 & 3782.96 \\
\hline 2.5 & 2.5 & 0.2 & 0.03311 & -273.261 & 1177.61 \\
\hline 2.5 & 2.5 & 0.6 & 0.04050 & -278.916 & 868.895 \\
\hline 2.5 & 2.5 & 1.5 & 0.03761 & 0.00355 & 2.3443 \\
\hline 2.5 & 2.5 & 3.0 & 0.03094 & 0.33237 & 2.64818 \\
\hline 2.5 & 2.5 & 6.0 & 0.02295 & 0.62511 & 3.25548 \\
\hline 2.5 & 2.5 & 10.0 & 0.01744 & 0.81623 & 3.85719 \\
\hline & & & & & \\
\hline
\end{tabular}


Conflicts of Interest: The author declares that there are no conflicts of interest regarding the publication of this paper.

\section{References}

[1] Z. Iqbal, A. Saboor, M. Ahmad, A note on Libby-Novick Kumaraswamy distribution. Presented in 15th ISSOS conference (2017).

[2] D. L. Libby, M. R. Novick, Multivariate generalized beta distributions with applications to utility assessment. J. Educ. Stat. 7(4) (1982), 271-294.

[3] P. Kumaraswamy, A generalized probability density function for double-bounded random processes. J. Hydrol. 46(1-2) (1980), 79-88.

[4] M. C. Jones, Kumaraswamy's distribution: A beta-type distribution with some tractability advantages. Stat. Methodol. 6(1) (2009), 70-81.

[5] J. McDonald, Some generalized functions for the size distribution of income. Econometrica. 52(3) (1984), 647-665.

[6] P. A. Mitnik, New properties of the Kumaraswamy distribution. Commun. Stat., Theory Meth. 42(5) (2013), 741-755.

[7] J. J. Chen, M. R. Novick, Bayesian analysis for binomial models with generalized beta prior distributions. J. Educ. Stat. 9(2) (1984), 163-175.

[8] M. M. Ristić, B. V. Popović, S. Nadarajah, Libby and Novick's generalized beta exponential distribution. J. Stat. Comput. Simul. 85(4) (2013), 740-761.

[9] G. M. Cordeiro, L. H. de Santana, E. M. Ortega, R. R. Pescim, A new family of distributions: LibbyNovick beta. Int. J. Stat. Probab. 3(2) (2014), 63.

[10]M. Ali Ahmed, The new form Libby-Novick distribution, Communications in Statistics - Theory and Methods. 50 (2021), 540.

[11] M. Rashid, Z. Iqbal, M. Hanif, Characterizations and entropy measures of the libby-novick generalized beta distribution. Adv. Appl. Stat. 63(2) (2020), 235-259

[12]Z. Iqbal, M. Rashid, M. Hanif, Properties of the Libby-Novick generalized beta distribution with application. Int. J. Anal. Appl. 19 (2021), 360-388.

[13] G. M. Cordeiro, R. dos Santos Brito, The beta power distribution. Brazil. J. Probab. Stat. 26(1) (2012), 88112.

[14] R. Dasgupta, On the distribution of burr with applications. Sankhya B, 73 (2011), 1-19. 